\title{
A Proof of the Weierstraß Gap Theorem not Using the Riemann-Roch Formula
}

\author{
Dedicated to our good friend George Andrews at the occasion of his 80th \\ birthday
}

Peter Paule and Cristian-Silviu Radu

\begin{abstract}
Usually, the Weierstraß gap theorem is derived as a straightforward corollary of the Riemann-Roch theorem. Our main objective in this article is to prove the Weierstraß gap theorem by following an alternative approach based on "first principles", which does not use the RiemannRoch formula. Having mostly applications in connection with modular functions in mind, we describe our approach for the case when the given compact Riemann surface is associated with the modular curve $X_{0}(N)$.
\end{abstract}

Mathematics Subject Classification. Primary 14H55, 11F03;

Secondary 11P83.

Keywords. Weierstraß gap theorem, Modular functions.

\section{Main Objective}

Various topical areas in the theory of partitions, such as congruences for partition numbers, are connected to modular functions for congruence subgroups of $\mathrm{SL}_{2}(\mathbb{Z})$ as, for instance, $\Gamma_{0}(N)$; see Sect. 15 for definitions. Such functions live on compact Riemann surfaces, for instance, on $X_{0}(N)$ for $\Gamma_{0}(N)$. Number theoretic aspects then relate to properties of certain subalgebras formed by these functions. In cases where the genus of such surfaces is zero like, for instance, for $X_{0}(5)$ and $X_{0}(7)$, these algebras essentially have a relatively simple structure. For positive genus $g$, for example, in the case of $X_{0}(11)$, this changes. One explanation is this: when considering sets of meromorphic functions with poles only at one point $p$, the Weierstraß gap theorem says that one can obtain functions with all possible pole orders at $p$ with exactly $g$ exceptions.

Theorem 1.1 (Weierstraß gap theorem; e.g., Sect. III.5.3 in [6]). Let $X$ be a compact Riemann surface having genus $g \geq 1$. Then, for each $p \in X$, there 
are precisely $g$ integers $n_{j}=n_{j}(p)$ with

$$
1=n_{1}<\cdots<n_{g} \leq 2 g-1,
$$

such that there does not exist a meromorphic function on $X$ which is holomorphic on $X \backslash\{p\}$ and which has a pole of pole order $n_{j}$ at $p$.

We want to stress that "precisely" in the theorem means that for any positive integer $n$ other than the $g$ values $n_{j}$, a meromorphic function with a pole of order $n$ at $p$ exists.

Usually, as in [6, III. 5.3], this theorem is derived as a straightforward corollary of the Riemann-Roch theorem. Our main objective in this article is to prove the Weierstraß gap theorem by following an alternative approach based on "first principles" which does not use the Riemann-Roch formula. Having mostly applications in connection with modular functions in mind, we describe our approach for the case when the given compact Riemann surface $X$ is associated with $X_{0}(N)$. Some ingredients of our setting are related to ideas from the celebrated paper [3] by Dedekind and Weber; see [2] for an English translation together with an excellent introduction by John Stillwell.

\section{Introduction}

To exemplify the usage of Weierstraß's gap theorem, we choose an example related to the classical Ramanujan congruences, which in further details are discussed in [14]. Following the definition given in Sect. 15, let

$$
M(N):=\text { field of meromorphic modular functions for } \Gamma_{0}(N) .
$$

To keep this article as much self-contained as possible, we list basic definitions and properties of modular functions in a separate Appendix Sect. 15.

One standard way to construct modular functions is by eta quotients, i.e., products of the form:

$$
\prod_{d \mid m} \eta(\mathrm{d} \tau)^{r_{d}}, \tau \in \mathbb{H}
$$

Here, $\mathbb{H}$ denotes the upper half of the complex plane, $m \in \mathbb{Z}_{>0}, r_{d}$ are chosen integers, and $\eta$ denotes the Dedekind eta function defined as

$$
\eta(\tau)=q(\tau / 24) \prod_{n=1}^{\infty}\left(1-q(\tau)^{n}\right) \quad \text { where } q(\tau)=\exp (2 \pi i \tau) .
$$

Usually one writes $q$ instead of $q(\tau)$.

The case $m=\ell, \ell \geq 5$ a prime, gives rise to a simple but important class of eta quotients:

$$
z_{\ell}(\tau):=\left(\frac{\eta(\ell \tau)}{\eta(\tau)}\right)^{\frac{24}{\operatorname{gcd}(\ell-1,24)}}
$$

which are modular functions in $M(\ell)$ with (e.g., [9, Chap. 7, Theorem 1])

$$
\operatorname{ord}_{[\infty]_{\ell}} z_{\ell}^{*}=\frac{\ell-1}{\operatorname{gcd}(\ell-1,12)} .
$$


Here, the notation $z_{\ell}^{*}$ is explained by the fact that, in general, every modular function $f \in M(N)$ gives rise to an induced meromorphic function $f^{*}: X_{0}(N) \rightarrow$ $\hat{\mathbb{C}}:=\mathbb{C} \cup\{\infty\}$ which for $x=[\tau]_{N}$ is defined as

$$
f^{*}(x)=f^{*}\left([\tau]_{N}\right):=f(\tau), \tau \in \hat{\mathbb{H}}:=\mathbb{H} \cup \mathbb{Q} \cup\{\infty\} ;
$$

see Sect. 15. There one also finds the definition of $[\tau]_{N}$ as the orbit of $\tau$ under $\Gamma_{0}(N)$, as well as definitions of basic notions like of $\operatorname{ord}_{[a / c]_{N}} f^{*}$, the order of $f^{*}$ at a cusp $[a / c]_{N}, a / c \in \mathbb{Q} \cup\{\infty\}$. Note that $[\infty]_{N}=[1 / 0]_{N}$.

Example 2.1 [9, Chap. 7, Theorem 1]. Consider

$$
z_{5}(\tau)=\left(\frac{\eta(5 \tau)}{\eta(\tau)}\right)^{6}=q \prod_{j=1}^{\infty}\left(\frac{1-q^{5 j}}{1-q^{j}}\right)^{6}=q+6 q^{2}+27 q^{3}+98 q^{4}+\cdots .
$$

We have $\operatorname{ord}_{[\infty]_{N}} f^{*}:=\operatorname{ord}_{q} f$, confirming that

$$
\operatorname{ord}_{[\infty]_{5}} z_{5}^{*}=\frac{5-1}{\operatorname{gcd}(5-1,12)}=1=\operatorname{ord}_{q} z_{5} .
$$

Because of

$$
\begin{gathered}
z_{\ell}\left(-\frac{1}{\tau}\right) z_{\ell}\left(\frac{\tau}{\ell}\right)=\ell^{-\frac{12}{\operatorname{gcd}(\ell-1,12)}, \ell \text { a prime } \geq 5,} \\
z_{5}\left(-\frac{1}{\tau}\right)=\frac{5^{-3}}{z_{5}(\tau / 5)}=\frac{1}{5^{3}}\left(\frac{1}{q^{1 / 5}}-6+9 q^{1 / 5}+10 q^{2 / 5}-\cdots\right),
\end{gathered}
$$

which owing to $\operatorname{ord}_{[0]_{N}} f^{*}:=\operatorname{ord}_{q^{1 / N}} f(-1 / \tau)$ confirms that

$$
\operatorname{ord}_{[0]_{5}} z_{5}^{*}=-\frac{5-1}{\operatorname{gcd}(5-1,12)}=-1=\operatorname{ord}_{q^{1 / 5}} z_{5}\left(-\frac{1}{\tau}\right) \text {. }
$$

In general, for $\ell$ a prime, $X_{0}(\ell)$ has exactly two cusps $[\infty]_{\ell}$ and $[0]_{\ell}$ with widths 1 and $\ell$, respectively; see [9, Chap. 2, Sect. 2], resp. Sect. 15 for the definition of width. The $q$-series (2.5) and (2.8) are the local $q$-expansions of $z_{5}^{*}$ at these cusps.

Being meromorphic, modular functions form fields. For example, a classical fact, e.g., [5, Proposition 7.5.1], is that $M(N)=\mathbb{C}(j(\tau), j(N \tau))$, where $j$ is the modular invariant (the Klein $j$ function). The subset

$$
\begin{aligned}
M^{!}(N):= & \left\{f \in M(N): f^{*} \text { has poles only at (finitely many) points }[\tau]_{N}\right. \\
& \text { with } \tau \in \mathbb{Q} \cup\{\infty\}\}
\end{aligned}
$$

obviously is not a field but a $\mathbb{C}$-algebra. ${ }^{1}$

Example 2.2. By definition (2.5) together with (2.6) and (2.9), $z_{5} \in M^{!}(5)$, because $z_{5}^{*}$ has its only pole of pole order 1 at $[0]_{5}$.

\footnotetext{
${ }^{1} \mathrm{~A} \mathbb{C}$-algebra is a commutative ring with 1 which is also a vector space over $\mathbb{C}$.
} 
An important $\mathbb{C}$-subalgebra, in particular, with regard to algorithms, is $M^{\infty}(N):=\left\{f \in M^{!}(N): f^{*}\right.$ has poles only at $\left.[\infty]_{N}\right\}$.

By [15, Lemma 20], $M^{\infty}(N)$ for each $N \geq 1$ contains an eta quotient $\mu_{N}$ of the form as in (2.1), such that $\operatorname{ord}_{[a / c]_{N}} \mu_{N}^{*}>0$ for all $a / c \in \mathbb{Q}$ with $[a / c]_{N} \neq[\infty]_{N}$. Hence, one can multiply with a suitable power of $\mu_{N}$ to turn any given $f \in M^{!}(N)$ into an element $\mu_{N}^{\alpha} f$ in $M^{\infty}(N)$.

Example 2.3. Choose $f(\tau):=j(\tau) \in M^{!}(N)$, the Klein $j$ function, and $\alpha$, such that $\mu_{N}^{\alpha} j \in M^{\infty}(N)$. Let $\beta:=\operatorname{ord}_{[\infty]_{N}} \mu_{N}$, then $\operatorname{ord}_{[\infty]_{N}} \mu_{N}^{\alpha} j=\alpha \beta-1$. In particular

$$
\operatorname{gcd}\left(\operatorname{ord}_{[\infty]_{N}} \mu_{N}, \operatorname{ord}_{[\infty]_{N}} \mu_{N}^{\alpha} j\right)=1
$$

which will be needed later. A description of how to construct such $\mu_{N}$ is given in $[15]$.

Since we will prove the gap theorem in the version of Theorem 12.2, where $X=X_{0}(N)$, and with $p=[\infty]_{N}$, a key issue in our approach concerns the question of finding appropriate representations of $M^{\infty}(N)$.

Example 2.4. Owing to (2.6) and (2.9), $1 / z_{5}=\frac{1}{q}-6+9 q+\cdots \in M^{\infty}(5)$. Because of $\operatorname{ord}_{[\infty]_{5}}\left(1 / z_{5}\right)^{*}=-1$, each $f \in M^{\infty}(5)$ can be written as a polynomial in $1 / z_{5}$; in short:

$$
M^{\infty}(5)=\mathbb{C}\left[\frac{1}{z_{5}}\right],
$$

where $\mathbb{C}[x]$ denotes the ring of polynomials in $x$ with complex coefficients. One also has

$$
M^{\infty}(7)=\mathbb{C}\left[\frac{1}{z_{7}}\right]
$$

but already for $\ell=11$, the situation is quite different. For example, in [14], we proved (implicitly) that $M^{\infty}(11)$ can be represented as a $\mathbb{C}\left[1 / z_{11}\right]$-module which is freely generated by modular functions $F_{2}, F_{3}, F_{4}$, and $F_{6} \in M^{\infty}(11)$. More concretely

$$
\begin{aligned}
M^{\infty}(11)=\langle 1 & \left.F_{2}, F_{3}, F_{4}, F_{6}\right\rangle_{\mathbb{C}\left[\frac{1}{z_{11}}\right]} \\
:= & \left\{p_{0}\left(z_{11}\right)+p_{2}\left(z_{11}\right) F_{2}+p_{3}\left(z_{11}\right) F_{3}\right. \\
& \left.\quad+p_{4}\left(z_{11}\right) F_{4}+p_{6}\left(z_{11}\right) F_{6}: p_{i}\left(z_{11}\right) \in \mathbb{C}\left[1 / z_{11}\right]\right\},
\end{aligned}
$$

where the $F_{i}$ are determined as follows [14, Sect. 9]: from the two functions $f_{2}, f_{3} \in M^{!}(22)$ :

$$
f_{2}(\tau):=q^{-2} \prod_{n=1}^{\infty} \frac{\left(1-q^{n}\right)\left(1-q^{2 n}\right)^{3}}{\left(1-q^{11 n}\right)^{3}\left(1-q^{22 n}\right)}
$$

and

$$
f_{3}(\tau):=q^{-3} \prod_{n=1}^{\infty} \frac{\left(1-q^{n}\right)^{3}\left(1-q^{2 n}\right)}{\left(1-q^{11 n}\right)\left(1-q^{22 n}\right)^{3}}
$$


one constructs the desired $F_{i} \in M^{\infty}(11)$ by

$$
\begin{aligned}
& F_{2}(\tau):=f_{2}(\tau)-\left(U_{2} f_{3}\right)(\tau)=q^{-2}+2 q^{-1}-12+5 q+8 q^{2}+\cdots, \\
& F_{3}(\tau):=f_{3}(\tau)-4\left(U_{2} f_{2}\right)(\tau)=q^{-3}-3 q^{-2}-5 q^{-1}+24-13 q-\cdots, \\
& F_{4}(\tau):=f_{2}(\tau)^{2}+\frac{1}{2}\left(U_{2} f_{3}^{2}\right)(\tau)=q^{-4}-\frac{3}{2} q^{-3}-\frac{7}{2} q^{-2}-\frac{21}{2} q^{-1}+48-\cdots, \\
& F_{6}(\tau):=f_{3}(\tau)^{2}+8\left(U_{2} f_{2}^{2}\right)(\tau)=q^{-6}-6 q^{-5}+7 q^{-4}+22 q^{-3}-41 q^{-2}+\cdots,
\end{aligned}
$$

where $U_{2}$ is the special case $\ell=2$ ("summing the even part") of the standard $U$-operator:

$$
U_{\ell} \sum_{k=N}^{\infty} a(k) q^{k}:=\sum_{k=\lceil N / \ell\rceil}^{\infty} a(\ell k) q^{k} .
$$

In addition to $\operatorname{ord}_{[\infty]_{11}}\left(1 / z_{11}\right)^{*}=-5$, one has

$$
\left(\operatorname{ord}_{[\infty]_{11}} F_{2}^{*}, \operatorname{ord}_{[\infty]_{11}} F_{3}^{*}, \operatorname{ord}_{[\infty]_{11}} F_{4}^{*}, \operatorname{ord}_{[\infty]_{11}} F_{6}^{*}\right)=(-2,-3,-4,-6) .
$$

Thus the minimal pole order of the functions which in the sense of (2.10) generate $M^{\infty}(11)$ is 2 , not 1 . Indeed, the gap at 1 is predicted by the Weierstraß gap theorem, Theorem 1.1, owing to the fact that the compact Riemann surface $X:=X_{0}(11)$ has genus 1. A formula for the genus of $X_{0}(N)$, if $N=\ell$ is a prime, for instance, can be found in [5, Exercises 3.1.4(e)]; the genus for general $N$ is determined in [5, Sect. 3.9].

In Sect. 12, we prove Theorem 12.2, a version of the gap Theorem 1.1 for the case $X=X_{0}(N)$ and with the only pole put at $\infty$, utilizing only first principles and avoiding the use of the Riemann-Roch formula. In particular, we avoid the use of any differentials. In addition, our approach provides new algebraic insight by consisting in a combination of module presentations of modular function algebras, integral bases, Puiseux series, and discriminants. For example, using our approach to prove the bound $\leq 2 g-1$ stated in the Weierstraß gap theorem is reduced to an elementary combinatorial argument, see Sect. 12. Another by-product of our proof of the Weierstraß gap Theorem 12.2 is a natural explanation of the genus $g=0$ case as a consequence of the reduction to an integral basis.

In view of various constructive aspects involved, we are planning to exploit the algorithmic content of our approach for computer algebra applications, for instance, for the effective computation of suitable module bases for modular functions. As already mentioned, some ideas we used trace back to the celebrated work [3] by Dedekind and Weber; see [2] for an English translation together with an excellent introduction by John Stillwell.

Finally, we remark that the history of Weierstraß's gap theorem and related topics such as Weierstraß points somehow presents a challenge. The historical account [4] by Andrea Del Centina describes the scientific evolution of the gap theorem up to the 1970s. Concerning its beginnings Centina says, "The history of Weierstraß points is not marked by a precise starting date 
because it is not clear when Weierstraß stated and proved his Lückensatz (or "gap" theorem), but one can argue that probably it was in the early 1860s."

The rest of our article is structured as follows. In Sect. 3, we introduce order-complete bases of modules over a polynomial ring $\mathbb{C}[t]$ to describe modular function algebras. In Sect. 4, we describe how such bases can be stepwise modified to obtain an integral basis; i.e., an order-complete basis for the full algebra $M^{\infty}(N)$. Under particular circumstances, one can keep track of the total number of such steps, which then gives a proof of the Weierstraß gap Theorem 12.2. To do this bookkeeping, one can use "order-reduction" polynomials discussed in Sect. 5. In Sect. 6, we explain how to obtain order-reduction polynomials computationally; Sect. 7 deals with important special cases. In Sects. 8 and 9, we derive important ingredients of our proof of Theorem 12.2; for example, a factorization property of the discriminant polynomial in Proposition 9.3. In Sects. 10 and 11, we relate discriminant polynomials to orderreduction polynomials associated with integral bases. In Sect. 12, we use these results to prove the Weierstraß gap theorem in the version of Theorem 12.2. To prove the bound $2 g-1$ for the size of the maximal gap, our approach allows a purely combinatorial argument (a gap property of monoids) which we describe in Sect. 13. At various places, we require functions to have the separation property, as defined in Sect. 9. In Sect. 14, we prove the existence of such functions by giving an explicit construction.

The first Appendix Sect. 15 gives a short account on basic modular function facts needed; the second Appendix Sect. 16 recollects some fundamental facts about meromorphic functions on Riemann surfaces.

\section{Modular Function Algebras as $\mathbb{C}[t]$-Modules}

We already used (implicitly) the convention that if a meromorphic function $f$ has a pole, then the pole order is defined as the negative order at this point, that is

$$
\operatorname{pord}_{p} f:=-\operatorname{ord}_{p} f .
$$

If $f \in M^{\infty}(N)$, we simplify notation using the convention for the pole order at infinity:

$$
\text { pord } f:=-\operatorname{ord}_{[\infty]_{N}} f^{*} .
$$

Definition 3.1. A tuple $\left(b_{0}, b_{1}, \ldots, b_{n-1}\right), n \geq 1$, of modular functions in $M^{\infty}(N)$ is called order-complete if

$$
b_{0}=1 \text { and } \operatorname{pord} b_{i} \equiv i \quad(\bmod n) \text { for } i=1, \ldots, n-1 .
$$

Slightly more generally, any tuple $\left(1, \beta_{1}, \ldots, \beta_{n-1}\right)$ which is a reordering of an order-complete tuple $\left(1, b_{1}, \ldots, b_{n-1}\right)$, that is,

$$
\left\{\beta_{1}, \ldots, \beta_{n-1}\right\}=\left\{b_{1}, \ldots, b_{n-1}\right\},
$$

is also called order-complete. 
Example 3.2. The tuple $\left(1, F_{6}, F_{2}, F_{3}, F_{4}\right)$ with $F_{j} \in M^{\infty}(11)$ as in $(2.10)$ is order-complete.

Example 3.3. Let

$$
f(\tau):=q \frac{1}{z_{11}} \prod_{k=1}^{\infty}\left(1-q^{11 k}\right) \sum_{n=0}^{\infty} p(11 n+6) q^{n} .
$$

The tuple $\left(1, f, f^{2}, f^{3}, f^{4}\right)$ is order-complete. Notice that pord $f=4$. In [13], it is shown that the subalgebra $\mathbb{C}\left[1 / z_{11}, f\right]$ of $M^{\infty}(11)$, which is generated by all bivariate polynomials in $1 / z_{11}$ and $f$, has a representation as a $\mathbb{C}\left[1 / z_{11}\right]$ module as follows:

$$
\mathbb{C}\left[\frac{1}{z_{11}}, f\right]=\left\langle 1, f, f^{2}, f^{3}, f^{4}\right\rangle_{\mathbb{C}\left[\frac{1}{z_{11}}\right]} .
$$

In view of these examples, we note that in contrast to $(2.10), \mathbb{C}\left[1 / z_{11}, f\right] \neq$ $M^{\infty}(11)$. For instance, it is obvious that this subalgebra does not contain any $g \in M^{\infty}(11)$ with pord $g=3$. Nevertheless, both function tuples

$$
\left\langle 1, F_{6}, F_{2}, F_{3}, F_{4}\right\rangle_{\mathbb{C}[t]}, \text { and }\left\langle 1, f, f^{2}, f^{3}, f^{4}\right\rangle_{\mathbb{C}[t]},
$$

form a basis of the corresponding $\mathbb{C}[t]$-module they generate, where $t:=1 / z_{11}$. Namely, since the generators have different pole-order modulo pord $t=5$, each element contained in these modules can be represented as a unique linear combination of the module generators with coefficients being polynomials in $t$. This motivates the following definition.

Definition 3.4. For $t \in M^{\infty}(N)$, let $n:=\operatorname{pord} t \geq 1$. Let, $M$ be the $\mathbb{C}[t]$ module generated by an order-complete tuple in $M^{\infty}(N)$, that is,

$$
\begin{aligned}
M & =\left\langle 1, b_{1}, b_{2}, \ldots, b_{n-1}\right\rangle_{\mathbb{C}[t]} \\
& :=\left\{p_{0}(t)+p_{1}(t) b_{1}+\cdots+p_{n-1}(t) b_{n-1}: p_{i}(x) \in \mathbb{C}[x]\right\} .
\end{aligned}
$$

Then, we call $\left(1, b_{1}, \ldots, b_{n-1}\right)$ an order-complete basis for $M$ over $\mathbb{C}[t]$. Slightly more generally, any tuple $\left(1, \beta_{1}, \ldots, \beta_{n-1}\right)$ which is a reordering, in the sense of $(3.1)$, of an order-complete basis $\left(1, b_{1}, \ldots, b_{n-1}\right)$ for $M$ is also called an order-complete basis for $M$.

Proposition 3.5. Let $t, f \in M^{\infty}(N)$ with $n:=\operatorname{pord} t \geq 1$ and $\operatorname{gcd}(n, \operatorname{pord} f)=$ 1. Then

$$
\mathbb{C}[t, f]=\left\langle 1, f, f^{2}, \ldots, f^{n-1}\right\rangle_{\mathbb{C}[t]},
$$

where $\left(1, f, f^{2}, \ldots, f^{n-1}\right)$ is an order-complete module basis.

Proof. If pord $f^{i} \equiv \operatorname{pord} f^{j}(\bmod n)$, then $n \mid(i-j)$ pord $f$. This implies $\{1,2, \ldots, n-1\}=\left\{\operatorname{pord} f(\bmod n), \operatorname{pord} f^{2}(\bmod n), \ldots, \operatorname{pord} f^{n-1}(\bmod n)\right\}$.

In addition, as a consequence of Theorem 7.1 and Lemma 7.3 in [14], $f^{n} \in$ $\left\langle 1, f, f^{2}, \ldots, f^{n-1}\right\rangle_{\mathbb{C}[t]}$. Hence, $\mathbb{C}[t, f] \subseteq\left\langle 1, f, f^{2}, \ldots, f^{n-1}\right\rangle_{\mathbb{C}[t]}$. The reverse direction of this inclusion is trivial, which completes the proof. 


\section{Integral Bases}

In Example 3.3, we saw that $\left(1, f, \ldots, f^{4}\right)$ is an order-complete basis of $\mathbb{C}\left[1 / z_{11}, f\right]$ which is a proper subalgebra of $M^{\infty}(11) .^{2}$ In this section, we shall see how such an order-complete basis can be step-wise modified to obtain an order-complete basis for the full algebra $M^{\infty}(11)$.

Definition 4.1. Let $t \in M^{\infty}(N)$ with $n:=$ pord $t \geq 1$. An order-complete tuple $\left(1, b_{1}, \ldots, b_{n-1}\right), b_{j} \in M^{\infty}(N)$ is called an integral basis for $M^{\infty}(N)$ over $\mathbb{C}[t]$ if

$$
\left\langle 1, b_{1}, \ldots, b_{n-1}\right\rangle_{\mathbb{C}[t]}=M^{\infty}(N)
$$

The motivation for this terminology comes from

Lemma 4.2. Let $f \in M(N)$ and $t \in M^{\infty}(N)$ with pord $t \geq 1$ and

$$
\operatorname{gcd}(\operatorname{pord} f, \operatorname{pord} t)=1 \text {. }
$$

Then, $f$ satisfies an algebraic relation

$$
f^{n}+p_{1}(t) f^{n-1}+\cdots+p_{n}(t)=0
$$

with polynomials $p_{j}(x) \in \mathbb{C}[x]$ (i.e., $f$ is integral over $\mathbb{C}[t]$ ) if and only if

$$
f \in M^{\infty}(N) \text {. }
$$

Moreover, if $f \in M^{\infty}(N)$, then there exists an algebraic relation with $n=$ pord $t$.

Proof. The statement with the assumption $f \in M^{\infty}(N)$ follows immediately from Proposition 3.5. For the other direction, assume that $m:=\operatorname{pord}_{p} f^{*}>0$ for $p \neq[\infty]_{N}$. Then $\operatorname{pord}_{p}\left(f^{n}\right)^{*}=m n$, a contradiction to

$$
\operatorname{pord}_{p}\left(p_{1}(t) f^{n-1}+\cdots+p_{n}(t)\right)^{*} \leq(n-1) m .
$$

A crucial observation for the process to obtain an integral basis for $M^{\infty}(N)$ from an order-complete basis is stated in the following.

Proposition 4.3. Let $t \in M^{\infty}(N)$ with $n:=\operatorname{pord} t \geq 1$. Let $\left(1, b_{1}, \ldots, b_{n-1}\right)$ with $b_{j} \in M^{\infty}(N)$ be an order-complete basis of the $\mathbb{C}[t]$-module:

$$
M:=\left\langle 1, b_{1}, \ldots, b_{n-1}\right\rangle_{\mathbb{C}[t]} \subseteq M^{\infty}(N) .
$$

Then, for any $f \in M^{\infty}(N)$, there exist polynomials $q(x)$ and $p_{j}(x)$ in $\mathbb{C}[x]$, such that

$$
f=\frac{p_{0}(t)}{q(t)}+\frac{p_{1}(t)}{q(t)} b_{1}+\cdots+\frac{p_{n-1}(t)}{q(t)} b_{n-1} .
$$

\footnotetext{
${ }^{2}$ Notice that pord $1 / z_{11}=5$.
} 
Proof. For $j \in \mathbb{Z}_{\geq 0}$, consider the sets

$$
G_{j}:=\left\{t^{j} f-h: h \in M\right\} .
$$

For each $j \geq 0$, choose a non-zero $g_{j} \in G_{j}$, such that pord $g_{j}$ is minimal amongst all the elements in $G_{j}$. By construction, using the convention $n \mathbb{Z}_{\geq 0}:=$ $\left\{n k: k \in \mathbb{Z}_{\geq 0}\right\}$, we have for all $j \geq 0$ :

$$
\text { pord } g_{j} \notin S:=\left(0+n \mathbb{Z}_{\geq 0}\right) \cup\left(\operatorname{pord} b_{1}+n \mathbb{Z}_{\geq 0}\right) \cup \cdots \cup\left(\operatorname{pord} b_{n-1}+n \mathbb{Z}_{\geq 0}\right) \text {. }
$$

Obviously, $S$ is an additive submonoid of $\left(\mathbb{Z}_{\geq 0},+\right)$. Moreover, $\mathbb{Z}_{\geq 0} \backslash S$ has only finitely many elements; let $k$ be the maximal element in this set. Then there exist $c_{j} \in \mathbb{C}$, not all zero, such that

$$
c_{0} g_{0}+c_{1} g_{1}+\cdots+c_{k+1} g_{k+1}=0
$$

This is owing to the fact that equating the coefficients of non-positive powers in the $q$-expansions of both sides (which are functions in $M^{\infty}(N)$ ) gives $k+1$ equations in $k+2$ variables $c_{j}$. Hence, the dimension of the $\mathbb{C}$-vector space $G$, which is generated by all the $g_{j}, j \geq 0$, is bounded by $k+1$. Using $g_{j}:=t^{j} f-h_{j}$ with $h_{j} \in M,(4.2)$ rewrites into the form:

$$
\begin{aligned}
c_{0}(f & \left.-h_{0}\right)+c_{1}\left(t f-h_{1}\right)+\cdots+c_{k+1}\left(t^{k+1} f-h_{k+1}\right) \\
& =\left(c_{0}+c_{1} t+\cdots+c_{k+1} t^{k+1}\right) f-\left(c_{0} h_{0}+c_{1} h_{1}+\cdots+c_{k+1} h_{k+1}\right)=0 .
\end{aligned}
$$

The linear combination of $h_{j}$ is in $M$; hence, this gives the desired relation for $f$ with $q(t)=c_{0}+c_{1} t+\cdots+c_{k+1} t^{k+1}$.

Corollary 4.4. Let $t \in M^{\infty}(N)$ with $n:=\operatorname{pord} t \geq 1$. Let $\left(1, b_{1}, \ldots, b_{n-1}\right)$ with $b_{j} \in M^{\infty}(N)$ be an order-complete basis of the $\mathbb{C}[t]$-module:

$$
M:=\left\langle 1, b_{1}, \ldots, b_{n-1}\right\rangle_{\mathbb{C}[t]} \subseteq M^{\infty}(N) .
$$

If $M \neq M^{\infty}(N)$, then there exist $c_{j} \in \mathbb{C}$, not all zero, and $\alpha$ in $\mathbb{C}$, such that

$$
h_{\alpha}:=\frac{c_{0}+c_{1} b_{1}+\cdots+c_{n-1} b_{n-1}}{t-\alpha} \in M^{\infty}(N) \backslash M .
$$

In particular, there exists a uniquely determined $k \in\{1, \ldots, n-1\}$, such that

$$
\operatorname{pord} h_{\alpha}=\operatorname{pord} b_{k}-n \geq k \text { and } c_{k} \neq 0 \text {. }
$$

Proof. By Proposition 4.3, there exists an $f \in M^{\infty}(N) \backslash M$ of the form (4.1), such that $q(x) \nmid p_{i}(x)$ for some $i \in\{0, \ldots, n-1\}$. Hence, there exists $\alpha \in \mathbb{C}$, such that $x-\alpha \mid q(x)$, but $x-\alpha \nmid p_{i}(x)$. Consequently, $q(t) /(t-\alpha) \in M^{\infty}(N)$ and thus

$$
g:=f \frac{q(t)}{t-\alpha}=\frac{p_{0}(t)+p_{1}(t) b_{1}+\cdots+p_{n-1}(t) b_{n-1}}{t-\alpha} \in M^{\infty}(N) \backslash M .
$$

By division with remainder, there are polynomials $q_{j}(x) \in \mathbb{C}[x]$ and $c_{j} \in \mathbb{C}$, such that $p_{j}(x)=(x-\alpha) q_{j}(x)+c_{j}, j=0, \ldots, n-1$. Rewriting the representation of $g$ and noting that $c_{i} \neq 0$ proves the first part of the statement on $h_{\alpha}$. To prove (4.4), consider

$$
(t-\alpha) h_{\alpha}=c_{0}+c_{1} b_{1}+\cdots+c_{n-1} b_{n-1},
$$


which implies

$$
\operatorname{pord}\left(t h_{\alpha}\right)=n+\operatorname{pord} h_{\alpha}=\max _{\substack{1 \leq j \leq n-1, c_{j} \neq 0}}\left\{\operatorname{pord} b_{j}\right\}
$$

Let $k$ be the index for which pord $b_{k}$ becomes maximal with $c_{k} \neq 0$. Recalling pord $b_{j} \equiv j(\bmod n), j=1, \ldots, n$, proves pord $b_{k} \geq k+n$. Because otherwise pord $b_{k}=k$ which owing to the choice of $k$ would imply pord $b_{j}=j$ for all $j=1, \ldots, n$, and the given order-complete basis would be integral. This proves (4.4).

Corollary 4.4 motivates the following.

Definition 4.5. Let $M=\left\langle 1, b_{1}, \ldots, b_{n-1}\right\rangle_{\mathbb{C}[t]}$ and $h_{\alpha} \in M^{\infty}(N)$ be as in Corollary 4.4 ; i.e., $M \neq M^{\infty}(N)$ and pord $h_{\alpha}=$ pord $b_{k}-n \geq k$. The replacement

$$
\left(1, \ldots, b_{k-1}, b_{k}, b_{k+1}, \ldots\right) \rightarrow\left(1, \ldots, b_{k-1}, h_{\alpha}, b_{k+1}, \ldots\right)
$$

of $b_{k}$ by $h_{\alpha}$ is called a pole-order-reduction step associated with $\alpha \in \mathbb{C}$.

We summarize in the form of

Proposition 4.6. Let $t \in M^{\infty}(N)$ with $n:=\operatorname{pord} t \geq 1$. Let $\left(1, b_{1}, \ldots, b_{n-1}\right)$ with $b_{j} \in M^{\infty}(N)$ be an order-complete basis of the $\mathbb{C}[t]$-module:

$$
M:=\left\langle 1, b_{1}, \ldots, b_{n-1}\right\rangle_{\mathbb{C}[t]} \subseteq M^{\infty}(N) .
$$

If $M \neq M^{\infty}(N)$, then:

(i) By a finite sequence of pole-order-reduction steps the order-complete basis $\left(1, b_{1}, \ldots, b_{n-1}\right)$ can be transformed into an integral basis $\left(1, \beta_{1}, \ldots, \beta_{n-1}\right)$, such that

$$
\left\langle 1, \beta_{1}, \ldots, \beta_{n-1}\right\rangle_{\mathbb{C}[t]}=M^{\infty}(N)
$$

(ii) If $\left(1, \beta_{1}^{\prime}, \ldots, \beta_{n-1}^{\prime}\right)$ is any another integral basis, that is,

$$
\left\langle 1, \beta_{1}^{\prime}, \ldots, \beta_{n-1}^{\prime}\right\rangle_{\mathbb{C}[t]}=M^{\infty}(N)
$$

then

$$
\left\{\operatorname{pord} \beta_{1}, \ldots, \operatorname{pord} \beta_{n-1}\right\}=\left\{\operatorname{pord} \beta_{1}^{\prime}, \ldots, \operatorname{pord} \beta_{n-1}^{\prime}\right\} .
$$

Proof. The proof of part (i) is an immediate consequence of Corollary 4.4. Namely, owing to (4.4), each step reduces the pole order of one of the basis elements by $n$. This guarantees termination in finitely many steps. To prove (ii), without loss of generality, we can assume that pord $\beta_{j} \equiv \operatorname{pord} \beta_{j}^{\prime} \equiv j$ $(\bmod n)$ for all $j$. Suppose pord $\beta_{j} \neq \operatorname{pord} \beta_{j}^{\prime}$ for some $j \in\{1, \ldots, n-1\}$, i.e., pord $\beta_{j}^{\prime}=$ pord $\beta_{j}+k n$ with $k \geq 1$. However, this implies that $\beta_{j} \notin$ $\left\langle 1, \beta_{1}^{\prime}, \ldots, \beta_{n-1}^{\prime}\right\rangle_{\mathbb{C}[t]}$, because then, no element in this module can have the same pole order as $\beta_{j}$, a contradiction. 


\section{Order-Reduction Polynomials}

It was shown in the previous section that by applying a procedure using finitely many steps, any order-complete basis of a subalgebra of $M^{\infty}(N)$ can be extended to an integral basis of $M^{\infty}(N)$. Moreover, by (4.5), the pole orders of the integral basis functions are uniquely determined. It turns out that under particular circumstances, one can keep track of the number of order-reduction steps, which then gives a proof of the Weierstraß gap Theorem 12.2. To do this bookkeeping, one can use "order-reduction" polynomials. To our knowledge, for the first time such polynomials have been used by Dedekind and Weber [3], see [2] for Stillwell's translation into English.

Throughout this section, $t \in M^{\infty}(N)$ with $n:=$ pord $t \geq 1$ and $\left(1, b_{1}, \ldots\right.$, $\left.b_{n-1}\right)$ with $b_{j} \in M^{\infty}(N)$ is an order-complete basis of the $\mathbb{C}[t]$-module:

$$
M:=\left\langle 1, b_{1}, \ldots, b_{n-1}\right\rangle_{\mathbb{C}[t]} \subseteq M^{\infty}(N) .
$$

Owing to $t(\tau)=\infty$ if and only if $[\tau]_{N}=[\infty]_{N}, t$ is a holomorphic function on $\mathbb{H}$. Moreover, the induced function $t^{*}$, which is meromorphic on the compact Riemann surface $X_{0}(N)$, has a pole only at $[\infty]_{N}$.

Remark 5.1 (A basic notational convention). In general, every modular function $f \in M(N)$ gives rise to an induced meromorphic function $f^{*}: X_{0}(N) \rightarrow \hat{\mathbb{C}}$ which for $x=[\tau]_{N}$ is defined as

$$
f^{*}(x)=f^{*}\left([\tau]_{N}\right):=f(\tau), \tau \in \hat{\mathbb{H}}
$$

see Appendix Sect. 15. A central theme in what follows is to consider maps:

$$
f^{*} \circ\left(t^{*} \mid U\right)^{-1}: V \rightarrow \hat{\mathbb{C}}
$$

where $U \subseteq X_{0}(N)$ and $V \subseteq \mathbb{C}$ are open sets, such that

$$
t^{*}: U \rightarrow V \text { is bi-holomorphic. }
$$

Hence, for $v \in V$, the evaluations

$$
f^{*} \circ\left(t^{*} \mid U\right)^{-1}(v)=f^{*}\left(\left(t^{*} \mid U\right)^{-1}(v)\right)
$$

have to be interpreted in the sense of (5.1), i.e., interpreting $x=\left(t^{*} \mid U\right)^{-1}(v)$ as $x=[\tau]_{N}$ for some $\tau \in \hat{\mathbb{H}}$.

Depending on the context, we will freely move between considering $t$ as a function on $\mathbb{H}$, resp. $\hat{\mathbb{H}}$, and its induced version $t^{*}: X_{0}(N) \rightarrow \hat{\mathbb{C}}$.

Using the terminology explained in the Appendix Sect. 16, we assume that $v_{0} \in \mathbb{C}$ is not a branch point of $t^{*}$; in short, $v_{0} \notin \operatorname{BranchPts}\left(t^{*}\right)$. In this case, there are $n$ pairwise distinct points $x_{j}=\left[\tau_{j}\right]_{N} \in X_{0}(N)$ with $\tau_{j} \in \mathbb{H}$, such that

$$
t^{*-1}\left(v_{0}\right)=\left\{x_{1}, \ldots, x_{n}\right\} .
$$

In addition, there exists a neighborhood $V$ of $v_{0}$ and neighborhoods $U_{j}$ of $x_{j}$, such that

$$
t^{*-1}(V)=U_{1} \cup \cdots \cup U_{n},
$$


as a disjoint union of open sets, and such that for $j=1, \ldots, n$, the restricted functions

$$
t^{*} \mid U_{j}: U_{j} \rightarrow V
$$

are bi-holomorphic.

Let

$$
T_{j}:=\left(t^{*} \mid U_{j}\right)^{-1}: V \rightarrow U_{j}, j=1, \ldots, n
$$

Define

$$
D_{t}\left(1, b_{1}, \ldots, b_{n-1}\right): V \rightarrow \mathbb{C}
$$

by

$$
D_{t}\left(1, b_{1}, \ldots, b_{n-1}\right)(v):=\left|\begin{array}{cccc}
1 & 1 & \cdots & 1 \\
\left(b_{1}^{*} \circ T_{1}\right)(v) & \left(b_{1}^{*} \circ T_{2}\right)(v) & \cdots & \left(b_{1}^{*} \circ T_{n}\right)(v) \\
\vdots & \vdots & \ddots & \vdots \\
\left(b_{n-1}^{*} \circ T_{1}\right)(v) & \left(b_{n-1}^{*} \circ T_{2}\right)(v) & \cdots & \left(b_{n-1}^{*} \circ T_{n}\right)(v)
\end{array}\right|^{2} .
$$

Taking the square of the determinant guarantees that the expression on the right side is symmetric with respect to any permutation of $T_{1}, \ldots, T_{n}$. Consequently, $D_{t}\left(1, b_{1}, \ldots, b_{n-1}\right)$ is a holomorphic function on $V$. Carrying out the same construction on neighborhoods $V$ for all $v_{0} \in \mathbb{C} \backslash \operatorname{BranchPts}\left(t^{*}\right)$, and gluing the resulting functions $D_{t}\left(1, b_{1}, \ldots, b_{n-1}\right): V \rightarrow \mathbb{C}$ together, gives a global holomorphic function:

$$
D_{t}\left(1, b_{1}, \ldots, b_{n-1}\right): \mathbb{C} \backslash \operatorname{BranchPts}\left(t^{*}\right) \rightarrow \mathbb{C} .
$$

Using the same arguments as in the proof of Theorem 8.2 in [7], this function can be extended to a meromorphic function:

$$
D_{t}\left(1, b_{1}, \ldots, b_{n-1}\right): \hat{\mathbb{C}}:=\mathbb{C} \cup\{\infty\} \rightarrow \hat{\mathbb{C}}
$$

with $\infty$ as its only pole. Classical complex analysis tells that $\mathcal{M}(\hat{\mathbb{C}})=\mathbb{C}(z)$, i.e., the field of meromorphic functions on $\hat{\mathbb{C}}$ are rational functions with coefficients in $\mathbb{C}$. Hence, we have the following.

Lemma 5.2. The meromorphic function $D_{t}\left(1, b_{1}, \ldots, b_{n-1}\right)(v)$ constructed above is a polynomial function in $v$.

Definition 5.3. The polynomial $D_{t}\left(1, b_{1}, \ldots, b_{n-1}\right)(x) \in \mathbb{C}[x]$ is called orderreduction polynomial for the order-complete basis $\left(1, b_{1}, \ldots, b_{n-1}\right), b_{j} \in M^{\infty}$ $(N)$, of the $\mathbb{C}[t]$-module

$$
\left\langle 1, b_{1}, \ldots, b_{n-1}\right\rangle_{\mathbb{C}[t]} \subseteq M^{\infty}(N),
$$

where $t \in M^{\infty}(N)$ with $n:=\operatorname{pord} t \geq 1$.

Example 5.4. Taking

$$
t:=\frac{1}{z_{11}}=\frac{1}{q^{5}}-\frac{12}{q^{4}}+\frac{54}{q^{3}}-\frac{88}{q^{2}}-\frac{99}{q}+540-418 q-\cdots \in M^{\infty}
$$

and

$$
\left(1, b_{1}, \ldots, b_{4}\right):=\left(1, F_{2}, F_{3}, F_{4}, F_{6}\right),
$$


where $F_{j} \in M^{\infty}(11)$ are as in Example 2.4, one obtains

$$
D_{1 / z_{11}}\left(1, F_{2}, F_{3}, F_{4}, F_{6}\right)(x)=x^{4}\left(5^{5} 11^{6}-2 \cdot 3^{2} \cdot 439081 x+5^{5} x^{2}\right) .
$$

Example 5.5. Taking $t$ and the $b_{j}$ as in Example 5.4, one obtains

$$
D_{1 / z_{11}}\left(1, F_{2}, F_{4}^{2}, F_{4}, F_{6}\right)(x)=\left(11^{3}+x\right)^{2} D_{1 / z_{11}}\left(1, F_{2}, F_{3}, F_{4}, F_{6}\right)(x) .
$$

Remark 5.6. How such polynomials are computed is explained in Sect. 6 .

In Corollary 4.4, we proved that if $M \neq M^{\infty}(N)$, then there exist $c_{j} \in \mathbb{C}$, not all zero, and $v_{0}$ in $\mathbb{C}$, such that

$$
\frac{c_{0}+c_{1} b_{1}+\cdots+c_{n-1} b_{n-1}}{t-v_{0}} \in M^{\infty}(N) \backslash M .
$$

Recall that we denoted the $n$ pairwise distinct preimages of $v_{0}$ as follows:

$$
t^{*}\left(x_{1}\right)=t^{*}\left(\left[\tau_{1}\right]_{N}\right)=t\left(\tau_{1}\right)=v_{0}, \ldots, t^{*}\left(x_{n}\right)=t^{*}\left(\left[\tau_{n}\right]_{N}\right)=t\left(\tau_{n}\right)=v_{0} .
$$

Relation (5.6) implies

$c_{0}+c_{1} b_{1}\left(\tau_{1}\right)+\cdots+c_{n-1} b_{n-1}\left(\tau_{1}\right)=0, \ldots, c_{0}+c_{1} b_{1}\left(\tau_{n}\right)+\cdots+c_{n-1} b_{n-1}\left(\tau_{n}\right)=0$.

As a necessary condition for the existence of $c_{j} \in \mathbb{C}$ not all zero, the determinant

$$
\left|\begin{array}{cccc}
1 & b_{1}\left(\tau_{1}\right) & \cdots & b_{n-1}\left(\tau_{1}\right) \\
1 & b_{1}\left(\tau_{2}\right) & \cdots & b_{n-1}\left(\tau_{2}\right) \\
\vdots & \vdots & \ddots & \vdots \\
1 & b_{1}\left(\tau_{n}\right) & \cdots & b_{n-1}\left(\tau_{n}\right)
\end{array}\right|
$$

of the corresponding linear system has to be zero. In view of

$$
\left(b_{i}^{*} \circ T_{j}\right)\left(v_{0}\right)=b_{i}^{*}\left(\left(t^{*} \mid U_{j}\right)^{-1}\left(v_{0}\right)\right)=b_{i}^{*}\left(x_{j}\right)=b_{i}\left(\tau_{j}\right),
$$

the square of this determinant (taking the underlying matrix transposed) is $D_{t}\left(1, b_{1}, \ldots, b_{n-1}\right)\left(v_{0}\right)$. Above, we used the fact that the definition for

$$
D_{t}\left(1, b_{1}, \ldots, b_{n-1}\right): \mathbb{C} \backslash \operatorname{BranchPts}\left(t^{*}\right) \rightarrow \mathbb{C}
$$

extends to the polynomial function

$$
D_{t}\left(1, b_{1}, \ldots, b_{n-1}\right): \hat{\mathbb{C}} \rightarrow \hat{\mathbb{C}} .
$$

This means, the case when $v_{0} \in \mathbb{C}$ is a branch point of $t^{*}$ is also covered by the same determinant condition:

$$
D_{t}\left(1, b_{1}, \ldots, b_{n-1}\right)\left(v_{0}\right)=0 .
$$

However, if $v_{0} \in \mathbb{C}$ is a branch point, this condition is automatically satisfied, because then at least the two rows

$$
\left(1, b_{1}\left(\tau_{i}\right), \ldots, b_{n-1}\left(\tau_{i}\right)\right) \text { and }\left(1, b_{1}\left(\tau_{j}\right), \ldots, b_{n-1}\left(\tau_{j}\right)\right),
$$

are equal for $i \neq j$. Summarizing, this gives 
Lemma 5.7. Let $t \in M^{\infty}(N)$ with $n:=$ pord $t \geq 1$. Let $\left(1, b_{1}, \ldots, b_{n-1}\right)$ with $b_{j} \in M^{\infty}(N)$ be an order-complete basis of the $\mathbb{C}[t]$-module

$$
M:=\left\langle 1, b_{1}, \ldots, b_{n-1}\right\rangle_{\mathbb{C}[t]} \subseteq M^{\infty}(N) \text { and } M \neq M^{\infty}(N) .
$$

Let $v_{0} \in \mathbb{C}$ be such that ${ }^{3}$

$$
\frac{c_{0}+c_{1} b_{1}+\cdots+c_{n-1} b_{n-1}}{t-v_{0}} \in M^{\infty}(N) \backslash M .
$$

for $c_{j} \in \mathbb{C}$, not all zero. Then

$$
D_{t}\left(1, b_{1}, \ldots, b_{n-1}\right)\left(v_{0}\right)=0 .
$$

If $v_{0}$ is a branch point of $t^{*}$, the condition (5.7) is automatically satisfied.

\section{How to Compute Order-Reduction Polynomials}

Next, we explain how to compute the order-reduction polynomials in (5.4) and (5.5).

To this end, it will be convenient to introduce the following notation:

Definition 6.1. If

$$
f(\tau)=\sum_{n=-K}^{\infty} f_{n} q^{n}
$$

is the $q$-expansion at infinity for some $f \in M^{\infty}(N)$, we define

$$
\tilde{f}(q):=\sum_{n=-K}^{\infty} f_{n} q^{n},
$$

that is,

$$
f(\tau)=\tilde{f}(q(\tau))=\tilde{f}(q) \text { with } q=q(\tau)=e^{2 \pi i \tau} \text { for } \tau \in \mathbb{H} .
$$

Returning to the setting (5.2), we again assume that $v_{0} \in \mathbb{C}$ is not a branch point of $t^{*}$. This means that there exists pairwise distinct $x_{j}=\left[\tau_{j}\right]_{N} \in$ $X_{0}(N)$ with $\tau_{j} \in \mathbb{H} \cup \mathbb{Q}$ such that $\left[\tau_{j}\right]_{N} \neq[\infty]_{N}$ and $^{4}$

$$
t^{*-1}\left(v_{0}\right)=\left\{x_{1}, \ldots, x_{n}\right\},
$$

together with neighborhoods $U_{j}$ of the $x_{j}$, such that for a suitable neighborhood $V$ of $v_{0}$ :

$$
t^{*-1}(V)=U_{1} \cup \cdots \cup U_{n},
$$

as a disjoint union of open sets, and such that the restricted functions

$$
T_{j}=\left(t^{*} \mid U_{j}\right)^{-1}: V \rightarrow U_{j}
$$

are bi-holomorphic.

\footnotetext{
${ }^{3}$ The existence of such a $v_{0}$ is owing to Corollary 4.4.

${ }^{4}$ Notice that, in particular, $\tau \neq \infty$.
} 
For each $j=1, \ldots, n$ and $v \in V$ our goal, achieved in Lemma 6.2(ii), is to determine expressions for $q_{j}(v):=q^{2 \pi i \tau(j)}$, where $\tau(j)$ is close to $\tau_{j}$, such that

$$
t^{*}\left([\tau(j)]_{N}\right)=t(\tau(j))=\tilde{t}\left(q_{j}(v)\right)=v .
$$

For $q=e^{2 \pi i \tau}$ with $\tau \in \mathbb{H}$, we have

$$
\tilde{t}(q)=\frac{1}{q^{n}}(1+\varphi(q)):=\frac{1}{q^{n}}\left(1+\varphi_{1} q+\varphi_{2} q^{2}+\cdots\right) .
$$

Here, we assume that the first coefficient in this $q$-expansion of $t$ is 1 . Now, if

$$
1+\psi(q):=1+\psi_{1} q+\psi_{2} q^{2}+\cdots:=\frac{1}{1+\varphi(q)},
$$

and

$$
(1+\psi(q))^{1 / n}:=\sum_{l=0}^{\infty}\left(\begin{array}{c}
1 / n \\
l
\end{array}\right) \psi(q)^{l}
$$

then

$$
\tilde{t}(q)=\frac{1}{U(q)^{n}}, \text { where } U(q):=q(1+\psi(q))^{1 / n} .
$$

To fix a branch of the $n$th root, we choose the preimage $\tau_{n}$ and recall that

$$
v_{0}=t^{*}\left(\left[\tau_{n}\right]_{N}\right)=t\left(\tau_{n}\right)=\tilde{t}\left(e^{2 \pi i \tau_{n}}\right) .
$$

Now, for each $v \in \mathbb{C}$ close to $v_{0}$, there is for each $j \in\{1, \ldots, n\}$ a uniquely determined $\tau(j) \in \mathbb{H}$ close to $\tau_{j}$, such that

$$
v=t^{*}\left([\tau(j)]_{N}\right)=t(\tau(j))=\tilde{t}\left(e^{2 \pi i \tau(j)}\right) .
$$

By choosing a neighborhood of $\tau_{n}$, we fix a branch of the $n$th root of $v \in \mathbb{C}$ close to $v_{0}$ :

$$
\sqrt[n]{v}:=\frac{1}{U(q)} \text { with } q=e^{2 \pi i \tau(n)},
$$

where $\tau(n)$ is close to $\tau_{n}$ and determined as in (6.3).

In addition, let $W$ be such that $U(W(q))=W(U(q))=q$, and define

$$
\zeta_{n}:=e^{\frac{2 \pi i}{n}}
$$

After this preparation, in view of (6.4) we can put things together as follows.

Lemma 6.2. In the given setting, for $j=1, \ldots, n$ and $v \in \mathbb{C}$ close to $v_{0}$, let

$$
q_{j}(v):=W\left(\zeta_{n}^{j} \frac{1}{\sqrt[n]{v}}\right)
$$

where $\sqrt[n]{v}$ is defined as in (6.4).

Then, for $j=1, \ldots, n$ and $v \in \mathbb{C}$ close to $v_{0}$ :

$$
q_{j}(v)=e^{2 \pi i \tau(j)},
$$

where $[\tau(j)]_{N}=T_{j}(v)$ with $\tau(j)$ as in (6.3), and

$$
\tilde{t}\left(q_{j}(v)\right)=v
$$

where the values $q_{j}(v)$ are pairwise distinct for $j=1, \ldots, n$. 
Proof. The values $q_{j}(v), j=1, \ldots, n$, are defined by power series in $q=$ $q(\tau(n))$ :

$q_{j}(v)=W\left(\zeta_{n}^{j} U(q)\right)=\zeta_{n}^{j} q+O\left(q^{2}\right)$ with $q=e^{2 \pi i \tau(n)}$ where $\tau(n)$ is close to $\tau_{n}$.

For a fixed $v$ close to $v_{0}$, these values are pairwise different for $j=1, \ldots, n$, because

$$
q_{j}(v)=W\left(\zeta_{n}^{j} U(q)\right)=W\left(\zeta_{n}^{k} U(q)\right)=q_{k}(v) \Rightarrow \zeta_{n}^{j} U(q)=\zeta_{n}^{k} U(q) .
$$

By $(6.2)$

$$
\tilde{t}\left(q_{j}(v)\right)=\frac{1}{U\left(q_{j}(v)\right)^{n}}=U\left(W\left(\zeta_{n}^{j} \frac{1}{\sqrt[n]{v}}\right)\right)^{-n}=v
$$

This implies (i) and (ii).

Lemma 6.2 enables us to compute the polynomial $D_{t}\left(1, b_{1}, \ldots, b_{n-1}\right)(v)$, because by part (i) with $i=1, \ldots, n-1$ and $j=1, \ldots, n$ :

$$
\left(b_{i}^{*} \circ T_{j}\right)(v)=b_{i}^{*}\left(T_{j}(v)\right)=\widetilde{b_{i}}\left(e^{2 \pi i \tau(j)}\right)=\sum_{\ell=-\operatorname{pord} b_{i}} \beta_{\ell}^{(i)} q_{j}(v)^{\ell}=\widetilde{b_{i}}\left(W\left(\zeta_{n}^{j} \frac{1}{\sqrt[n]{v}}\right)\right) .
$$

This means, each $\left(b_{i}^{*} \circ T_{j}\right)(v)$ can be represented as a Laurent series in powers of $1 / v^{1 / n}$ :

$$
\left(b_{i}^{*} \circ T_{j}\right)(v)=\zeta_{n}^{-j \operatorname{pord} b_{i}} v^{\frac{\operatorname{pord} b_{i}}{n}}+\alpha_{i, j} v^{\frac{\operatorname{pord} b_{i}-1}{n}}+\cdots+\beta_{i, j} \frac{1}{v^{1 / n}}+\cdots,
$$

with coefficients $\alpha_{i, j}, \beta_{i, j}$, etc., in $\mathbb{C}$, and under the assumption that the first Laurent series coefficient $\beta_{- \text {pord } b_{i}}^{(i)}$ of each $\widetilde{b_{i}}\left(q_{j}(v)\right)$ is equal to 1 . Owing to Lemma $5.2, D_{t}\left(1, b_{1}, \ldots, b_{n-1}\right)(v)$ as defined in $(5.3)$ must be a polynomial in $v$. Consequently, we can compute it by taking suitable truncated versions of the expansions (6.5).

Remark 6.3. This is how we computed the order-reduction polynomials in (5.4) and (5.5).

\section{Discriminant Polynomials}

Important special cases of order-reduction polynomials are produced by ordercomplete module bases of $\mathbb{C}[t, f]$ of the form as in Proposition 3.5.

Definition 7.1. Let $t, f \in M^{\infty}(N)$ with $n:=\operatorname{pord} t \geq 1$, and $\operatorname{gcd}(n, \operatorname{pord} f)=$ 1. Then

$$
D_{t}(f)(v):=D_{t}\left(1, f, f^{2}, \ldots, f^{n-1}\right)(v)
$$

is called the discriminant polynomial for the order-complete basis $(1, f, \ldots$, $f^{n-1}$ ) of the $\mathbb{C}[t]$-module:

$$
\left\langle 1, f, f^{2} \ldots, f^{n-1}\right\rangle_{\mathbb{C}[t]}=\mathbb{C}[t, f] \subseteq M^{\infty}(N) .
$$


The discriminant polynomial

$$
\begin{aligned}
D_{t}(f)(v) & =\left|\begin{array}{cccc}
1 & 1 & \cdots & 1 \\
f^{*}\left(T_{1}(v)\right) & f^{*}\left(T_{2}(v)\right) & \cdots & f^{*}\left(T_{n}(v)\right) \\
\vdots & \vdots & \ddots & \vdots \\
f^{*}\left(T_{1}(v)\right)^{n-1} & f^{*}\left(T_{2}(v)\right)^{n-1} & \cdots & f\left(T_{n}(v)\right)^{n-1}
\end{array}\right|^{2} \\
& =\prod_{1 \leq i<j \leq n}\left(f^{*}\left(T_{i}(v)\right)-f^{*}\left(T_{j}(v)\right)\right)^{2}
\end{aligned}
$$

factors as the square of a Vandermonde determinant. Now, invoking (6.5) with $b_{i}=f$, and thus, pord $b_{i}=$ pord $f$, gives

$$
f^{*}\left(T_{j}(v)\right)=\zeta_{n}^{-j \operatorname{pord} f} v^{\frac{\text { pord } f}{n}}+\alpha_{j} v^{\frac{\operatorname{pord} f-1}{n}}+\cdots+\beta_{j} \frac{1}{v^{1 / n}}+\cdots .
$$

Hence

$$
f^{*}\left(T_{i}(v)\right)-f^{*}\left(T_{j}(v)\right)=\left(\zeta_{n}^{-i \operatorname{pord} f}-\zeta_{n}^{-j \operatorname{pord} f}\right) v^{\frac{\operatorname{pord} f}{n}}+\cdots
$$

and thus

$$
D_{t}(f)(v)=\text { constant } \cdot v^{2\left(\begin{array}{c}
n \\
2
\end{array}\right) \frac{\operatorname{pord} f}{n}}+\cdots .
$$

Summarizing, we have the following.

Lemma 7.2. Let $t, f \in M^{\infty}(N)$ with $n:=\operatorname{pord} t \geq 1$, and $\operatorname{gcd}(n$, pord $f)=1$. Then, the degree of the discriminant polynomial $D_{t}(f)(x) \in \mathbb{C}[x]$ is

$$
\operatorname{deg}_{x} D_{t}(f)(x)=(n-1) \text { pord } f .
$$

\section{Reduction Steps and Order-Reduction Polynomials}

In Sect. 4, we described how order-complete bases can be transformed into integral bases of $M^{\infty}(N)$ by a finite sequence of pole-order-reduction steps. In this section, we establish a link between pole-order-reduction steps and order-reduction polynomials.

To this end, we consider again our standard situation: let $t \in M^{\infty}(N)$ with $n:=$ pord $t \geq 1$, let $\left(1, b_{1}, \ldots, b_{n-1}\right)$ with $b_{j} \in M^{\infty}(N)$ be an ordercomplete basis of the $\mathbb{C}[t]$-module:

$$
M:=\left\langle 1, b_{1}, \ldots, b_{n-1}\right\rangle_{\mathbb{C}[t]} \subseteq M^{\infty}(N) .
$$

By Corollary 4.4, when $M \neq M^{\infty}(N)$, there exist $c_{j} \in \mathbb{C}$, not all zero, and $\alpha$ in $\mathbb{C}$, such that

$$
h_{\alpha}:=\frac{c_{0}+c_{1} b_{1}+\cdots+c_{n-1} b_{n-1}}{t-\alpha} \in M^{\infty}(N) \backslash M .
$$

In particular, there exists a $k \in\{1, \ldots, n-1\}$, such that

$$
\text { pord } h_{\alpha}=\operatorname{pord} b_{k}-n \geq k \text { and } c_{k} \neq 0 \text {. }
$$


Proposition 8.1. With regard to order-reduction polynomials, this setting is reflected by

$$
\begin{aligned}
D_{t} & \left(1, b_{1}, \ldots, b_{k-1}, h_{\alpha}, b_{k+1}, \ldots, b_{n-1}\right)(v) \\
& =\frac{c_{k}^{2}}{(v-\alpha)^{2}} D_{t}\left(1, b_{1}, \ldots, b_{k-1}, b_{k}, b_{k+1}, \ldots, b_{n-1}\right)(v) .
\end{aligned}
$$

Proof. After filling the right side of (8.1) into the determinant definition (5.3) of $D_{t}\left(1, b_{1}, \ldots, b_{k-1}, h_{\alpha}, b_{k+1}, \ldots, b_{n-1}\right)(v)$ and noticing that $t^{*}\left(T_{j}(v)\right)=v$, $j=1, \ldots, n$, the proof is a straightforward consequence of determinant calculus.

In other words, a pole-order-reduction step associated with $\alpha \in \mathbb{C}$ :

$$
\left(1, \ldots, b_{k-1}, b_{k}, b_{k+1}, \ldots\right) \rightarrow\left(1, \ldots, b_{k-1}, h_{\alpha}, b_{k+1}, \ldots\right),
$$

from one order-complete basis to another corresponds to factoring the orderreduction polynomial as

$$
\begin{aligned}
& D_{t}\left(1, b_{1}, \ldots, b_{k-1}, b_{k}, b_{k+1}, \ldots, b_{n-1}\right)(x) \\
& \quad=\text { constant } \cdot(x-\alpha)^{2} D_{t}\left(1, b_{1}, \ldots, b_{k-1}, h_{\alpha}, b_{k+1}, \ldots, b_{n-1}\right)(x) .
\end{aligned}
$$

Example 8.2. In the situation of Example 5.5

$$
F_{3}+12 F_{2}+11^{2}=-\frac{161,051+15,972 F_{2}+F_{4}^{2}+242 F_{4}-121 / 4 F_{6}}{1 / z_{11}+11^{3}} .
$$

\section{Local Puiseux Expansions}

By considering local expansions at finitely many points $\left[\tau_{j}\right]_{N} \in X_{0}(N)$ for $\tau_{j} \in \hat{\mathbb{H}}=\mathbb{H} \cup \mathbb{Q} \cup\{\infty\}$, in this section, we derive important ingredients for our proof of Theorem 12.2. To this end, we consider charts $\varphi_{\tau_{0}}: U_{0} \rightarrow \mathbb{C}$ with $\varphi_{\tau_{0}}\left([\tau]_{N}\right):=\phi_{\tau_{0}}(\tau)$ defined in a standard way either by

$$
\phi_{\tau_{0}}(\tau):=\tau-\tau_{0}
$$

if $\tau_{0} \in \mathbb{H}$ is not an elliptic point, or by

$$
\phi_{\tau_{0}}(\tau):=\left(\frac{\tau-\tau_{0}}{\tau-\overline{\tau_{0}}}\right)^{h\left(\tau_{0}\right)}
$$

if $\tau_{0} \in \mathbb{H}$ is an elliptic point (cf. (9.5)), or according to (15.3) by

$$
\phi_{\tau_{0}}(\tau):=e^{2 \pi i \gamma^{-1} \tau / w_{N}(c)}
$$

if $\tau_{0}=\frac{a}{c}=\gamma \infty \in \mathbb{Q} \cup\{\infty\}$.

Here, $U_{0} \subseteq X_{0}(N)$ is a neighborhood of $\left[\tau_{0}\right]_{N}$; furthermore, the periods $h\left(\tau_{0}\right)$ equal either 2 or 3 . We note explicitly that all these charts are centered at 0 , that is,

$$
\phi_{\tau_{0}}\left(\tau_{0}\right)=0
$$


Remark 9.1. The explanation why such charts have to be chosen can be found, for instance, in [5, Sect. 2.2 and Sect. 2.3]. Charts, being homeomorphisms between open subsets of Riemann surfaces and of $\mathbb{C}$, are used to set up local series expansions. Charts of the kind as in (9.2) have to be taken when $\left[\tau_{0}\right]_{N}$ is an elliptic point, i.e., if

$$
\left\{\gamma \in \Gamma_{0}(N): \gamma \tau_{0}=\tau_{0}\right\} \neq\left\{\left(\begin{array}{ll}
1 & 0 \\
0 & 1
\end{array}\right),\left(\begin{array}{rr}
-1 & 0 \\
0 & -1
\end{array}\right)\right\} .
$$

Throughout this section, again $t \in M^{\infty}(N)$ with $n:=\operatorname{pord} t \geq 1$. Now, we reconsider the setting in Sect. 5 by dropping the assumption that $v_{0} \in \mathbb{C}$ is not a branch point of $t^{*}$. This means, we allow $\ell \leq n$ pairwise distinct points $x_{j}=\left[\tau_{j}\right]_{N} \in X_{0}(N)$ with $\tau_{j} \in \mathbb{H} \cup \mathbb{Q}$, such that $\left[\tau_{j}\right]_{N} \neq[\infty]_{N}$ and $^{5}$

$$
t^{*-1}\left(v_{0}\right)=\left\{x_{1}, \ldots, x_{\ell}\right\}
$$

There exists a neighborhood $V_{0}$ of $v_{0}$ and neighborhoods $U_{j}$ of the $x_{j}$, such that

$$
t^{*-1}\left(V_{0}\right)=U_{1} \cup \cdots \cup U_{\ell},
$$

as a disjoint union of open sets.

Now, if $\ell<n$, not all of the restricted functions

$$
t^{*} \mid U_{j}: U_{j} \rightarrow V_{0}
$$

are bi-holomorphic.

Summarizing this setting,

$$
t^{*}(x)=v_{0} \text { has } \ell \leq n \text { solutions } x_{1}=\left[\tau_{1}\right]_{N}, \ldots, x_{\ell}=\left[\tau_{\ell}\right]_{N}
$$

with multiplicities $k_{1}, \ldots, k_{\ell}$, respectively, i.e., $k_{1}+\cdots+k_{\ell}=n$.

Hence, if $V$ is an open subset of $V_{0}$ not containing $v_{0}$, then for each $j=1, \ldots, \ell$, there exist pairwise disjoint open subsets $U_{j, k} \subseteq U_{j}, k=1, \ldots, k_{j}$, such that

$$
t^{*-1}(V)=\left(U_{1,1} \cup \cdots \cup U_{1, k_{1}}\right) \cup \cdots \cup\left(U_{\ell, 1} \cup \cdots \cup U_{\ell, k_{\ell}}\right)
$$

as a disjoint union, and for $k=1, \ldots, k_{j}$, the restricted functions

$$
t^{*} \mid U_{j, k}: U_{j, k} \rightarrow V
$$

are bi-holomorphic.

For all $[\tau]_{N} \in U_{j}, j=1, \ldots, \ell$, one has expansions

$$
t^{*}\left([\tau]_{N}\right)=v_{0}+a_{j, 0} \phi_{\tau_{j}}(\tau)^{k_{j}}+a_{j, 1} \phi_{\tau_{j}}(\tau)^{k_{j}+1}+\cdots \text { with } a_{j, 0} \neq 0 .
$$

Again, using (6.1), one has

$$
t(\tau)-v_{0}=B_{j}\left(\phi_{\tau_{j}}(\tau)\right)^{k_{j}},
$$

where

$$
B_{j}(z):=z\left(a_{j, 0}+a_{j, 1} z+\cdots\right)^{1 / k_{j}}
$$

${ }^{5}$ Note that, in particular, $\tau_{j} \neq \infty$. 
For $j=1, \ldots, \ell$, let

$$
A_{j}(z)=A_{j, 1} z+A_{j, 2} z^{2}+\cdots \text { such that } A_{j}\left(B_{j}(z)\right)=B_{j}\left(A_{j}(z)\right)=z .
$$

Now, by inverting the relation (9.8) and using the Puiseux series, the situation of (9.6) is reflected as follows: for each $v \in V$, there is for fixed $(j, k), j=$ $1, \ldots, k_{j}$ and $k \in\left\{1, \ldots, k_{j}\right\}$, a uniquely determined $\tau=\tau(j, k) \in U_{j, k}$, such that

$$
[\tau]_{N}=[\tau(j, k)]_{N}=\left(t^{*} \mid U_{j, k}\right)^{-1}(v) .
$$

For such pairs $\tau=\tau(j, k)$ and $v$, one has

$$
\begin{aligned}
\phi_{\tau_{j}}(\tau)=\phi_{\tau_{j}}(\tau(j, k)) & =A_{j}\left(\zeta_{k_{j}}^{k}\left(v-v_{0}\right)^{1 / k_{j}}\right) \\
& =A_{j, 1} \zeta_{k_{j}}^{k}\left(v-v_{0}\right)^{1 / k_{j}}+A_{j, 2} \zeta_{k_{j}}^{2 k}\left(v-v_{0}\right)^{2 / k_{j}}+\cdots
\end{aligned}
$$

As in Sect. 5, one works with a fixed branch of the $k_{j}$ th root; moreover, we note that as a consequence of the definition of $A_{j}(z), A_{j, 1} \neq 0$ for all $j=1, \ldots, \ell$.

To connect to discriminant polynomials, let $f \in M^{\infty}(N)$ be such that $\operatorname{gcd}(n, \operatorname{pord} f)=1$. Moreover, without loss of generality, for $j=1, \ldots, \ell$, we can assume that the neighborhoods $U_{j}$ are chosen, such that the following expansions exist for all $[\tau]_{N} \in U_{j}$ :

$$
f^{*}\left([\tau]_{N}\right)=f(\tau)=f\left(\tau_{j}\right)+\sum_{m=1}^{\infty} b_{j, m} \phi_{\tau_{j}}(\tau)^{m} .
$$

Invoking (9.9), one obtains

Lemma 9.2. For $v_{0} \in \mathbb{C}$ and $j=1, \ldots, \ell$, suppose that open neighborhoods $U_{j, k}, k=1, \ldots, k_{j}$ and $V$ are chosen as above. Then, there exist series expansions with complex coefficients $c_{j, p}$, such that for all $v \in V$ :

$$
f\left(\left(t^{*} \mid U_{j, k}\right)^{-1}(v)\right)=f\left(\tau_{j}\right)+\sum_{p=1}^{\infty} c_{j, p} \zeta_{k_{j}}^{p k}\left(v-v_{0}\right)^{p / k_{j}}
$$

Proof. Setting $[\tau]_{N}:=\left(t^{*} \mid U_{j, k}\right)^{-1}(v) \in U_{j, k}$, the statement follows from applying (9.9) to $(9.10)$ :

$$
f(\tau)=f\left(\tau_{j}\right)+\sum_{m=1}^{\infty} b_{j, m}\left(A_{j, 1} \zeta_{k_{j}}^{k}\left(v-v_{0}\right)^{1 / k_{j}}+A_{j, 2} \zeta_{k_{j}}^{2 k}\left(v-v_{0}\right)^{2 / k_{j}}+\cdots\right)^{m} .
$$

To adapt to the refined setting (9.6), we extend our $T_{j}$-notation to the additional restricted functions:

$$
T_{j, k}=\left(t^{*} \mid U_{j, k}\right)^{-1}: V \rightarrow U_{j, k} .
$$

Finally, we use the information we obtained in terms of the local holomorphic Puiseux series expansion to represent the discriminant polynomial at $v_{0} \in \mathbb{C}$. 
Namely, for all $v \in V$ :

$$
\begin{aligned}
& D_{t}(f)(v)=(-1)^{\left(\begin{array}{c}
n \\
2
\end{array}\right)} \prod_{(j, k) \neq\left(j^{\prime}, k^{\prime}\right)}\left(f\left(T_{j, k}(v)\right)-f\left(T_{j^{\prime}, k^{\prime}}(v)\right)\right) \\
& =(-1)^{\left(\begin{array}{l}
n \\
2
\end{array}\right)} \prod_{\substack{1 \leq j \leq \ell \\
1 \leq k, k^{\prime} \leq k_{j}, k \neq k^{\prime}}}\left(f\left(T_{j, k}(v)\right)-f\left(T_{j, k^{\prime}}(v)\right)\right) \\
& \prod_{\substack{1 \leq j, j^{\prime} \leq \ell, j \neq j^{\prime} \\
1 \leq k, k^{\prime} \leq k_{j}}}\left(f\left(T_{j, k}(v)\right)-f\left(T_{j^{\prime}, k^{\prime}}(v)\right)\right) \\
& =(-1)^{\left(\begin{array}{c}
n \\
2
\end{array}\right)} \prod_{\substack{1 \leq j \leq \ell \\
1 \leq k, k^{\prime} \leq k_{j}, k \neq k^{\prime}}}\left(\sum_{p=1}^{\infty} c_{j, p}\left(\zeta_{k_{j}}^{p k}-\zeta_{k_{j}}^{p k^{\prime}}\right)\left(v-v_{0}\right)^{p / k_{j}}\right) \\
& \prod_{\substack{1 \leq j, j^{\prime} \leq \ell, j \neq j^{\prime} \\
1 \leq k, \bar{k}^{\prime} \leq k_{j}}}\left(f\left(\tau_{j}\right)-f\left(\tau_{j^{\prime}}\right)+O\left(\left(v-v_{0}\right)^{1 / k_{j}}\right)-O\left(\left(v-v_{0}\right)^{1 / k_{j^{\prime}}}\right)\right) .
\end{aligned}
$$

The last equality is by (9.11); it gives rise to the following.

Proposition 9.3. Let $t \in M^{\infty}(N)$ with $n=$ pord $t \geq 1$, let $f \in M^{\infty}(N)$ be such that $\operatorname{gcd}(n$, pord $f)=1$. For $v_{0} \in \mathbb{C}$, suppose that $t^{*}(x)=v_{0}$ has $\ell \leq n$ pairwise distinct solutions $x_{1}=\left[\tau_{1}\right]_{N}, \ldots, x_{\ell}=\left[\tau_{\ell}\right]_{N}$ with multiplicities $k_{1}, \ldots, k_{\ell}$, respectively, i.e., $k_{1}+\cdots+k_{\ell}=n$.

If

$$
\text { the values } f\left(\tau_{1}\right), \ldots, f\left(\tau_{\ell}\right) \text { are pairwise distinct, }
$$

and

$$
f^{\prime}\left(\tau_{1}\right) \neq 0, \ldots, f^{\prime}\left(\tau_{\ell}\right) \neq 0,
$$

then there exists a polynomial $p(x) \in \mathbb{C}[x]$, such that for all $v \in \mathbb{C}$ :

$$
D_{t}(f)(v)=\left(v-v_{0}\right)^{n-\ell} p(v) \text { where } p\left(v_{0}\right) \neq 0 .
$$

Proof. The statement follows from the last equality of the derivation preceding this proposition. Namely, under the condition (9.12), the second product on the right side of this equality is non-zero for $v=v_{0}$. Condition (9.13) means that $b_{j, 1} \neq 0$ in $(9.10)$, thus $c_{j, 1} \neq 0$ for $j=1, \ldots, \ell$ in $(9.11)$. Consequently, from the first product in the expression under consideration, one can pull out $v-v_{0}$ as follows:

$$
\begin{aligned}
& c_{1,1} \prod_{1 \leq k, k^{\prime} \leq k_{1}, k \neq k^{\prime}}\left(\zeta_{k_{1}}^{k}-\zeta_{k_{1}}^{k^{\prime}}\right)\left(v-v_{0}\right)^{1 / k_{1}} \cdots c_{\ell, 1} \prod_{1 \leq k, k^{\prime} \leq k_{\ell}, k \neq k^{\prime}}\left(\zeta_{k_{\ell}}^{k}-\zeta_{k_{\ell}}^{k^{\prime}}\right)\left(v-v_{0}\right)^{1 / k_{\ell}} \\
& =\text { constant } \cdot\left(v-v_{0}\right)^{2\left(\begin{array}{c}
k_{1} \\
2
\end{array}\right) \frac{1}{k_{1}}+\cdots+2\left(\begin{array}{c}
k_{\ell} \\
2
\end{array}\right) \frac{1}{k_{\ell}}}
\end{aligned}
$$

Recalling that $k_{1}+\cdots+k_{l}=n$ completes the proof for all $v \in V$, where $V$ is an open subset of a neighborhood $V_{0}$ of $v_{0}$, such that $V$ does not contain $v_{0}$. However, invoking the identity theorem from complex analysis, the statement extends to all $v \in \mathbb{C}$. 
Properties (9.12) and (9.13) are sufficiently important to deserve a

Definition 9.4 (separation property). Let $t \in M^{\infty}(N)$ with $n:=\operatorname{pord} t \geq 1$, let $f \in M^{\infty}(N)$ be such that $\operatorname{gcd}(n$, pord $f)=1$. For $v_{0} \in \mathbb{C}$, suppose that $t^{*}(x)=v_{0}$ has $\ell \leq n$ pairwise distinct solutions $x_{1}=\left[\tau_{1}\right]_{N}, \ldots, x_{\ell}=\left[\tau_{\ell}\right]_{N}$. We say that $f$ has the separation property for $\left(t, v_{0}\right)$ if $f$ satisfies $(9.12)$ and (9.13).

Remark 9.5. In Sect. 14, we describe how to construct such an $f$ having the separation property.

An immediate consequence of Proposition 9.3 is

Corollary 9.6. Let $f$ have the separation property for $(t, \beta)$ with $\beta \in \mathbb{C}$. Then

$$
D_{t}(f)(\beta)=0 \Longleftrightarrow \beta \in \operatorname{BranchPts}\left(t^{*}\right) .
$$

Another consequence of our analysis above is

Proposition 9.7. Let $t \in M^{\infty}(N)$ with $n:=\operatorname{pord} t \geq 1$, and let $f \in M^{\infty}(N)$ be such that $\operatorname{gcd}(n$, pord $f)=1$. For $v_{0} \in \mathbb{C}$, suppose that $t^{*}(x)=v_{0}$ has $\ell \leq n$ pairwise distinct solutions $x_{1}=\left[\tau_{1}\right]_{N}, \ldots, x_{\ell}=\left[\tau_{\ell}\right]_{N}$ with multiplicities $k_{1}, \ldots, k_{\ell}$, respectively, i.e., $k_{1}+\cdots+k_{\ell}=n$.

For complex numbers $a_{0}, \ldots, a_{n-1}$, not all zero, define a meromorphic function on $\mathbb{H}$ by

$$
F(\tau):=\frac{a_{0}+a_{1} f(\tau)+\cdots+a_{n-1} f(\tau)^{n-1}}{t(\tau)-v_{0}} .
$$

If $f$ has the separation property for $\left(t, v_{0}\right)$, then ${ }^{6}$

$$
F\left(\tau_{j}\right)=\infty \text { for some } j \in\{1, \ldots, \ell\} .
$$

Proof. Suppose $F^{*}$ is analytic on $X_{0}(N) \backslash\left\{[\infty]_{N}\right\}$. Then, assuming the setting as above, by (9.7), one has for all $\tau \in U_{1}$, a series expansion

$$
\begin{aligned}
a_{0} & +a_{1} f(\tau)+\cdots+a_{n-1} f(\tau)^{n-1}=\left(t(\tau)-v_{0}\right) F(\tau) \\
& =\left(a_{1,0} \phi_{\tau_{1}}(\tau)^{k_{1}}+a_{1,1} \phi_{\tau_{1}}(\tau)^{k_{1}+1}+\cdots\right)\left(F_{0}+F_{1} \phi_{\tau_{1}}(\tau)+\cdots\right)
\end{aligned}
$$

with $a_{1,0} \neq 0$. Hence, owing to (9.4),

$$
a_{0}+a_{1} f\left(\tau_{1}\right)+\cdots+a_{n-1} f\left(\tau_{1}\right)^{n-1}=0,
$$

which implies a factorization

$$
\begin{aligned}
a_{0} & +a_{1} f(\tau)+\cdots+a_{n-1} f(\tau)^{n-1} \\
& =\left(f(\tau)-f\left(\tau_{1}\right)\right)\left(A_{0}+A_{1} f(\tau)+\cdots+A_{n-2} f(\tau)^{n-2}\right) \\
& =\left(b_{1,0} \phi_{\tau_{1}}(\tau)+b_{1,1} \phi_{\tau_{1}}(\tau)^{2}+\cdots\right)\left(A_{0}+A_{1} f(\tau)+\cdots+A_{n-2} f(\tau)^{n-2}\right),
\end{aligned}
$$

$\overline{{ }^{6} \text { Note that } \tau_{j} \in} \mathbb{H} \cup \mathbb{Q}$ are such that $\left[\tau_{j}\right]_{N} \neq[\infty]_{N}$. Hence, (9.16) implies that $F \notin M^{\infty}(N)$. 
where the last equality is by (9.10) with $b_{1,0} \neq 0$ owing to (9.13). As a consequence of (9.17), if $k_{1}>1$ :

$$
A_{0}+A_{1} f\left(\tau_{1}\right)+\cdots+A_{n-2} f\left(\tau_{1}\right)^{n-2}=0,
$$

and by iteration

$$
\begin{aligned}
a_{0} & +a_{1} f(\tau)+\cdots+a_{n-1} f(\tau)^{n-1} \\
& =\left(f(\tau)-f\left(\tau_{1}\right)\right)^{k_{1}}\left(B_{0}+B_{1} f(\tau)+\cdots+B_{n-1-k_{1}} f(\tau)^{n-1-k_{1}}\right) .
\end{aligned}
$$

Notice that if

$$
B(x):=B_{0}+B_{1} x+\ldots+B_{n-1-k_{1}} x^{n-1-k_{1}}
$$

is the zero polynomial (e.g., if $k_{1}=n$ ), the assumption that $\left(1, f, \ldots, f^{n-1}\right.$ ) is an order-complete basis would imply that all $a_{j}=0$, and the proof would stop with this contradiction.

Using the same argument, one derives

$$
\begin{aligned}
a_{0} & +a_{1} f(\tau)+\cdots+a_{n-1} f(\tau)^{n-1} \\
& =\left(f(\tau)-f\left(\tau_{2}\right)\right)^{k_{2}}\left(C_{0}+C_{1} f(\tau)+\ldots+C_{n-1-k_{2}} f(\tau)^{n-1-k_{2}}\right),
\end{aligned}
$$

etc., up to

$$
\begin{aligned}
a_{0} & +a_{1} f(\tau)+\ldots+a_{n-1} f(\tau)^{n-1} \\
& =\left(f(\tau)-f\left(\tau_{\ell}\right)\right)^{k_{\ell}}\left(D_{0}+D_{1} f(\tau)+\ldots+D_{n-1-k_{\ell}} f(\tau)^{n-1-k_{\ell}}\right) .
\end{aligned}
$$

If one of the polynomial factors in the role of $B(x)$ above would be the zero polynomial, we are done. Otherwise, invoking condition (9.12) implies that

$$
\begin{gathered}
\left(f(\tau)-f\left(\tau_{1}\right)\right)^{k_{1}} \cdots\left(f(\tau)-f\left(\tau_{\ell}\right)\right)^{k_{\ell}} \text { divides } \\
a_{0}+a_{1} f(\tau)+\cdots+a_{n-1} f(\tau)^{n-1} .
\end{gathered}
$$

Recalling that not all the $a_{j}$ are zero and $k_{1}+\cdots+k_{\ell}=n$, we obtain a contradiction to the assumption that $F^{*}$ is analytic on $X_{0}(N) \backslash\left\{[\infty]_{N}\right\}$.

Corollary 9.8. Let $t \in M^{\infty}(N)$ with $n:=\operatorname{pord} t \geq 1$, let $f \in M^{\infty}(N)$ be such that $\operatorname{gcd}(n$, pord $f)=1$. For $v_{0} \in \mathbb{C}$, suppose that

$t^{*}(x)=v_{0}$ has $\ell \leq n$ pairwise distinct solutions $x_{1}=\left[\tau_{1}\right]_{N}, \ldots, x_{\ell}=\left[\tau_{\ell}\right]_{N}$.

For complex numbers $a_{0}, \ldots, a_{n-1}$, define a meromorphic function on $\mathbb{H}$ by

$$
F(\tau):=\frac{a_{0}+a_{1} f(\tau)+\cdots+a_{n-1} f(\tau)^{n-1}}{t(\tau)-v_{0}} .
$$

If $f$ has the separation property for $\left(t, v_{0}\right)$, then

$$
F \in M^{\infty}(N) \Longrightarrow F=0 \text {. }
$$

Proof. If one of the $a_{j}$ would be non-zero, Proposition 9.7 would imply a pole of $F^{*}$ at some $\left[\tau_{j}\right]_{N} \neq[\infty]_{N}, \tau_{j} \in \mathbb{H} \cup \mathbb{Q}$. 


\section{Order Reduction and Discriminant Polynomials}

In this section, we relate discriminant polynomials to order-reduction polynomials associated with integral bases. Throughout this section, let $t \in M^{\infty}(N)$ with $n:=$ pord $t \geq 1$, let $\left(1, b_{1}, \ldots, b_{n-1}\right), b_{j} \in M^{\infty}(N)$, be an order-complete tuple forming an integral basis for $M^{\infty}(N)$ over $\mathbb{C}[t]$, that is,

$$
\left\langle 1, b_{1}, \ldots, b_{n-1}\right\rangle_{\mathbb{C}[t]}=M^{\infty}(N) .
$$

Moreover, let $f \in M^{\infty}(N)$ again be chosen, such that $\operatorname{gcd}(n, \operatorname{pord} f)=1$. By Proposition 3.5, such an $f$ gives rise to an order-complete basis $\left(1, f, \ldots, f^{n-1}\right)$ of the $\mathbb{C}[t]$-module:

$$
\left\langle 1, f, f^{2}, \ldots, f^{n-1}\right\rangle_{\mathbb{C}[t]}=\mathbb{C}[t, f] \subseteq M^{\infty}(N) .
$$

By exemplifying the case for $n=3$, we shall see how the discriminant polynomial

$$
D_{t}(f)(v):=D_{t}\left(1, f, f^{2}, \ldots, f^{n-1}\right)(v)
$$

is related to the order-reduction polynomial:

$$
D_{t}\left(1, b_{1}, \ldots, b_{n-1}\right)(v) .
$$

By the identity theorem from complex analysis, it is sufficient to consider the situation for $v$ from a neighborhood $V$ of $v_{0} \in V$. With the setting as in (5.3), one has

$$
\begin{gathered}
\left(\begin{array}{ccc}
1 & 0 & 0 \\
r_{0}^{(1)}(v) & r_{1}^{(1)}(v) & r_{2}^{(1)}(v) \\
r_{0}^{(2)}(v) & r_{1}^{(2)}(v) & r_{2}^{(2)}(v)
\end{array}\right)\left(\begin{array}{ccc}
1 & 1 & 1 \\
\left(b_{1} \circ T_{1}\right)(v) & \left(b_{1} \circ T_{2}\right)(v) & \left(b_{1} \circ T_{3}\right)(v) \\
\left(b_{2} \circ T_{1}\right)(v) & \left(b_{2} \circ T_{2}\right)(v) & \left(b_{2} \circ T_{3}\right)(v)
\end{array}\right) \\
=\left(\begin{array}{ccc}
1 & 1 & 1 \\
f\left(T_{1}(v)\right) & f\left(T_{2}(v)\right) & f\left(T_{3}(v)\right) \\
f\left(T_{1}(v)\right)^{2} & f\left(T_{2}(v)\right)^{2} & f\left(T_{3}(v)\right)^{2}
\end{array}\right),
\end{gathered}
$$

because owing to (10.1), there exist polynomials $r_{j}^{(i)}(x) \in \mathbb{C}[x]$, such that

$$
f(\tau)^{i}=r_{0}^{(i)}(t(\tau))+r_{1}^{(i)}(t(\tau)) b_{1}(\tau)+r_{2}^{(i)}(t(\tau)) b_{2}(\tau), \tau \in \mathbb{H} .
$$

This implies

$$
f\left(T_{j}(v)\right)^{i}=r_{0}^{(i)}(v)+r_{1}^{(i)}(v)\left(b_{1} \circ T_{j}\right)(v)+r_{2}^{(i)}(v)\left(b_{2} \circ T_{j}\right)(v)
$$

using

$$
t\left(T_{j}(v)\right)=t\left(\left(t \mid U_{j}\right)^{-1}(v)\right)=v .
$$

Taking determinants of both sides of the matrix equation squared, this gives for the general case:

$$
\text { as polynomials in } v: D_{t}\left(1, b_{1}, \ldots, b_{n-1}\right)(v) \text { divides } D_{t}(f)(v) .
$$

Next, we consider the other direction. By Proposition 4.3, there exist polynomials $q_{j}(x)$ and $p_{i}(x)$ in $\mathbb{C}[x]$, such that

$$
b_{j}=\frac{p_{0}^{(j)}(t)}{q_{j}(t)}+\frac{p_{1}^{(j)}(t)}{q_{j}(t)} f+\cdots+\frac{p_{n-1}^{(j)}(t)}{q_{j}(t)} f^{n-1}, j=1, \ldots, n-1,
$$


where $q_{j}(t)$ is either a constant or such that

$$
\operatorname{gcd}\left(q_{j}(x), p_{0}^{(j)}(x), \ldots, p_{n-1}^{(j)}(x)\right)=1 .
$$

As before, this can be expressed as a matrix equation. We display the case for $n=3$ :

$$
\begin{gathered}
\left(\begin{array}{ccc}
1 & 0 & 0 \\
s_{0}^{(1)}(v) & s_{1}^{(1)}(v) & s_{2}^{(1)}(v) \\
s_{0}^{(2)}(v) & s_{1}^{(2)}(v) & s_{2}^{(2)}(v)
\end{array}\right)\left(\begin{array}{ccc}
1 & 1 & 1 \\
f\left(T_{1}(v)\right) & f\left(T_{2}(v)\right) & f\left(T_{3}(v)\right) \\
f\left(T_{1}(v)\right)^{2} & f\left(T_{2}(v)\right)^{2} & f\left(T_{3}(v)\right)^{2}
\end{array}\right) \\
=\left(\begin{array}{ccc}
1 & 1 & 1 \\
\left(b_{1} \circ T_{1}\right)(v) & \left(b_{1} \circ T_{2}\right)(v) & \left(b_{1} \circ T_{3}\right)(v) \\
\left(b_{2} \circ T_{1}\right)(v) & \left(b_{2} \circ T_{2}\right)(v) & \left(b_{2} \circ T_{3}\right)(v)
\end{array}\right),
\end{gathered}
$$

where

$$
s_{i}^{(j)}(v):=\frac{p_{i}^{(j)}(v)}{q_{j}(v)} .
$$

Again, taking determinants of both sides of the matrix equation squared, for the general case, this gives another polynomial relation in $v$ :

$$
D_{t}\left(1, b_{1}, \ldots, b_{n-1}\right)(v)=\frac{s(v)^{2}}{q_{1}(v)^{2} \cdots q_{n-1}(v)^{2}} D_{t}(f)(v),
$$

where $s(x), q_{1}(x), \ldots, q_{n-1}(x)$ are polynomials in $\mathbb{C}[x]$. It will be convenient to cancel out possible common factors and to write, as polynomials in $\mathbb{C}[x]$ :

$$
D_{t}\left(1, b_{1}, \ldots, b_{n-1}\right)(x)=\frac{S(x)^{2}}{Q_{1}(x)^{2} \cdots Q_{n-1}(x)^{2}} D_{t}(f)(x),
$$

such that

$S(x)$ and $Q_{1}(x) \cdots Q_{n-1}(x)$ are relatively prime polynomials, (10.6) and

$$
Q_{j}(x) \text { divides } q_{j}(x), j=1, \ldots, n-1,
$$

where $q_{j}(x)$ are determined as in (10.3).

\section{Order-Reduction Polynomials: Further Results}

In this section, we continue the considerations made in the previous section. Again, $t \in M^{\infty}(N)$ with $n:=$ pord $t \geq 1$, and $\left(1, b_{1}, \ldots, b_{n-1}\right)$ with $b_{j} \in$ $M^{\infty}(N)$ is assumed to be an integral basis for $M^{\infty}(N)$ over $\mathbb{C}[t]$.

Lemma 11.1. Let $\left(1, \beta_{1}, \ldots, \beta_{n-1}\right)$ with $\beta_{j} \in M^{\infty}(N)$ be an integral bases for $M^{\infty}(N)$ over $\mathbb{C}[t]$. Then, there exists a $c \in \mathbb{C}$ such that

$$
D_{t}\left(1, b_{1}, \ldots, b_{n-1}\right)(x)=c \cdot D_{t}\left(1, \beta_{1}, \ldots, \beta_{n-1}\right)(x) .
$$


Proof. Applying the same kind of argument as used to derive (10.2), we obtain the polynomial relations:

$$
D_{t}\left(1, b_{1}, \ldots, b_{n-1}\right)(x) \text { divides } D_{t}\left(1, \beta_{1}, \ldots, \beta_{n-1}\right)(x)
$$

and

$$
D_{t}\left(1, \beta_{1}, \ldots, \beta_{n-1}\right)(x) \text { divides } D_{t}\left(1, b_{1}, \ldots, b_{n-1}\right)(x) .
$$

This proves the statement.

Another application of the argument we used to derive (10.2) is the existence of some polynomial $R(x) \in \mathbb{C}[x]$, such that

$$
R(x)^{2} D_{t}\left(1, b_{1}, \ldots, b_{n-1}\right)(x)=D_{t}(f)(x) .
$$

This, using (10.5), implies

$$
\frac{R(x) S(x)}{Q_{1}(x) \cdots Q_{n-1}(x)}=1 \text { or }-1 .
$$

Finally, as a consequence of (10.6), we obtain

$$
R(x)=\frac{1}{c} \cdot Q_{1}(x) \cdots Q_{n-1}(x) \text { and } S(x)=c \text { for some non-zero } c \in \mathbb{C} .
$$

We summarize the following.

Lemma 11.2. There is $c \in \mathbb{C}$ such that

$$
D_{t}(f)(x)=c \cdot Q_{1}(x)^{2} \cdots Q_{n-1}(x)^{2} D_{t}\left(1, b_{1}, \ldots, b_{n-1}\right)(x),
$$

where for $j=1, \ldots, n-1$, the polynomials $Q_{j}(x)$ divide the polynomials $q_{j}(x)$ which are determined as in (10.3).

Lemma 11.3. Let $Q_{j}(x)$ be the polynomials as in Lemma 11.2. Suppose $f$ has the separation property for $(t, \beta)$ for some $\beta \in \mathbb{C}$. Then

$$
Q_{j}(\beta) \neq 0 \text { for all } j=1, \ldots, n-1 \text {. }
$$

Proof. Suppose $x-\beta \mid Q_{j}(x)$ for some $j \in\{1, \ldots, n-1\}$. By Lemma 11.2, $Q_{j}(x) \mid q_{j}(x)$ with $q_{j}(x)$ as in relation (10.3). Hence, $x-\beta$ divides $q_{j}(x)$, and (10.3) can be rewritten as

$$
\frac{q_{j}(t)}{t-\beta} b_{j}=\frac{p_{0}^{(j)}(t)}{t-\beta}+\frac{p_{1}^{(j)}(t)}{t-\beta} f+\cdots+\frac{p_{n-1}^{(j)}(t)}{t-\beta} f^{n-1} .
$$

As in the proof of Corollary 4.4, by division with remainder, there are polynomials $p_{l}(x) \in \mathbb{C}[x]$ and $a_{l} \in \mathbb{C}$, such that $p_{l}^{(j)}(x)=(x-\beta) p_{l}(x)+a_{l}$, $l=0, \ldots, n-1$. This means

$$
\begin{aligned}
\frac{q_{j}(t)}{t-\beta} b_{j}= & \frac{a_{0}+a_{1} f+\cdots+a_{n-1} f^{n-1}}{t-\beta} \\
& +p_{0}(t)+p_{1}(t) f+\cdots+p_{n-1}(t) f^{n-1} \in M^{\infty}(N) .
\end{aligned}
$$


Owing to the fact that $f$ has the separation property for $(t, \beta)$, one has by Corollary 9.8:

$$
\frac{a_{0}+a_{1} f+\cdots+a_{n-1} f^{n-1}}{t-\beta}=0 .
$$

Iterating this argument cancels out all powers of $t-\beta$ and one arrives at a representation of $b_{j}$ of the form:

$$
Q(t) b_{j}=P_{0}(t)+P_{1}(t) f+\cdots+P_{n-1}(t) f^{n-1}
$$

with polynomials $P_{l}(x)$ and $Q(x)$, such that

$$
x-\beta \nmid Q(x) .
$$

Comparing this to the representation (10.3), which rewrites as

$$
q_{j}(t) b_{j}=p_{0}^{(j)}(t)+p_{1}^{(j)}(t) f+\cdots+p_{n-1}^{(j)}(t) f^{n-1},
$$

produces a contradiction to the uniqueness of the basis representation since in contrast to (11.5), $x-\beta$ divides the denominator polynomial $q_{j}(x)$.

Proposition 11.4. For any $\beta \in \mathbb{C}$ :

$$
D_{t}\left(1, b_{1}, \ldots, b_{n-1}\right)(\beta)=0 \Longleftrightarrow \beta \in \operatorname{BranchPts}\left(t^{*}\right) .
$$

Proof. For the proof we choose $f$ having the separation property for $(t, \beta) .^{7}$ For the " $\Rightarrow$ " direction of the statement, suppose $D_{t}\left(1, b_{1}, \ldots, b_{n-1}\right)(\beta)=0$. Then (11.4) implies $D_{t}(f)(\beta)=0$ which, owing to Corollary 9.6, is true if and only if $\beta \in \operatorname{BranchPts}(t)$. For the other direction, we use the reverse direction of this "if and only if" relation: $\beta \in \operatorname{BranchPts}\left(t^{*}\right)$ implies $x-\beta \mid D_{t}(f)(x)$. Next, we apply Lemma 11.3 to the equation (10.5) and obtain

$$
x-\beta \mid D_{t}\left(1, b_{1}, \ldots, b_{n-1}\right)(x),
$$

which completes the proof.

From all this we obtain the complete factorization of order-reduction polynomials of integral bases. To state it, it is convenient to define

$$
\operatorname{BranchPts}_{\mathbb{C}}\left(t^{*}\right):=\operatorname{BranchPts}\left(t^{*}\right) \cap \mathbb{C},
$$

in order to keep the point $\infty$ out, as the image of the only pole at $[\infty]_{N}$.

Proposition 11.5. Let $t \in M^{\infty}(N)$ with $n:=$ pord $t \geq 1$ and $\left(1, b_{1}, \ldots, b_{n-1}\right)$ with $b_{j} \in M^{\infty}(N)$ be an integral basis for $M^{\infty}(N)$ over $\mathbb{C}[t]$. Then, there exists a $c \in \mathbb{C}$ and positive integers $m_{\beta}$, such that

$$
D_{t}\left(1, b_{1}, \ldots, b_{n-1}\right)(x)=c \cdot \prod_{\beta \in \operatorname{BranchPts}_{\mathbb{C}}\left(t^{*}\right)}(x-\beta)^{m_{\beta}} .
$$

Moreover, for any $\beta \in \operatorname{BranchPts}_{\mathbb{C}}\left(t^{*}\right)$, suppose that $t^{*}(x)=\beta$ has

$$
\ell(\beta)<n \text { pairwise distinct solutions } x_{1}^{(\beta)}=\left[\tau_{1}^{(\beta)}\right]_{N}, \ldots, x_{\ell(\beta)}^{(\beta)}=\left[\tau_{\ell(\beta)}^{(\beta)}\right]_{N}
$$

with multiplicities $k_{1}^{(\beta)}, \ldots, k_{\ell(\beta)}^{(\beta)}$, respectively, i.e., $k_{1}^{(\beta)}+\cdots+k_{\ell_{\beta}}^{(\beta)}=n$.

\footnotetext{
${ }^{7}$ How to construct such $f$ is described in Sect. 14 .
} 
Then

$$
m_{\beta}=n-\ell(\beta) .
$$

Proof. The factorization (11.7) is immediate from Proposition 11.4. To prove (11.8), let $\beta \in \operatorname{BranchPts}_{\mathbb{C}}\left(t^{*}\right)$ be a branch point of the kind as stated. Choose $f$ to have the separation property for $(t, \beta)$. Then (9.14) implies the existence of a polynomial $p(x) \in \mathbb{C}[x]$, such that

$$
D_{t}(f)(x)=(x-\beta)^{n-\ell(\beta)} p(x), \text { where } p(\beta) \neq 0 .
$$

According to (11.4), there exist polynomials $Q_{j}(x)$, such that

$$
D_{t}(f)(x)=c \cdot Q_{1}(x)^{2} \cdots Q_{n-1}(x)^{2} D_{t}\left(1, b_{1}, \ldots, b_{n-1}\right)(x)
$$

and owing to Lemma 11.3,

$$
Q_{j}(\beta) \neq 0 \text { for all } j=1, \ldots, n-1 \text {. }
$$

Hence, $(x-\beta)^{n-\ell(\beta)}$ divides $D_{t}\left(1, b_{1}, \ldots, b_{n-1}\right)(x)$ with the maximal power, which proves (11.8).

For the next consideration, we again have to use the charts as in (9.1), (9.2), and (9.3). In the setting of Proposition 11.5, one has

$$
\begin{aligned}
D_{t}\left(1, b_{1}, \ldots, b_{n-1}\right)(x) & =c \cdot \prod_{\beta \in \operatorname{BranchPts}_{\mathbb{C}}\left(t^{*}\right)}(x-\beta)^{k_{1}^{(\beta)}+\cdots+k_{\ell(\beta)}^{(\beta)}-\ell(\beta)} \\
& =c \cdot \prod_{\beta \in \operatorname{BranchPts}_{\mathbb{C}}\left(t^{*}\right)}(x-\beta)^{k_{1}^{(\beta)}-1} \cdots(x-\beta)^{k_{\ell(\beta)}^{(\beta)}-1} \\
& =c \cdot \prod_{\beta \in \operatorname{BranchPts}_{\mathbb{C}}\left(t^{*}\right)}\left(x-t\left(\tau_{1}^{(\beta)}\right)\right)^{k_{1}^{(\beta)}-1} \cdots\left(x-t\left(\tau_{\ell(\beta)}^{(\beta)}\right)\right)^{k_{\ell(\beta)}^{(\beta)}-1} \\
& =c \cdot \prod_{\beta \in \operatorname{BranchPts}_{\mathbb{C}}\left(t^{*}\right)} \prod_{j=1}^{\ell(\beta)}\left(x-t\left(\tau_{j}^{(\beta)}\right)\right)^{-1+\operatorname{mult}_{\left[\tau_{j}^{(\beta)}\right]_{N}}^{\left(\left(\tau^{*}\right)\right.}} \\
& =c \cdot \prod_{\substack{\text { all orbits } \left.\tau_{0}\right]_{N} \in X_{0}(N),\left[\tau_{0}\right]_{N} \neq[\infty]_{N}}}\left(x-t\left(\tau_{0}\right)\right)^{-1+\operatorname{mult}_{\left[\tau_{0}\right]_{N}}\left(\tau^{*}\right)},
\end{aligned}
$$

where the last line is by the fact that if $t\left(\tau_{0}\right) \notin \operatorname{BranchPts}\left(t^{*}\right)$, then

$$
-1+\operatorname{mult}_{\left[\tau_{0}\right]_{N}}\left(\tau^{*}\right)=0
$$

Here, we use the notion of multiplicity $\operatorname{mult}_{x}(f)$, also explained in Sect. 16, which stands for the multiplicity at the point $x \in X$ of a meromorphic function $f$ on a (compact) Riemann surface $X$. For $x_{0}=\left[\tau_{0}\right]_{N} \in X_{0}(N)$, one has (e.g., [11, Lemma 4.7] and [5, Sect. 2.4]) with respect to our charts $\phi_{\tau_{0}}(\tau)$ centered at $0:^{8}$

$$
\text { mult }_{x_{0}}\left(t^{*}\right)= \begin{cases}\operatorname{ord}_{\phi_{\tau_{0}}(\tau)}\left(t(\tau)-t\left(\tau_{0}\right)\right), & \text { if }\left[\tau_{0}\right]_{N} \text { is no pole of } t^{*}, \\ -\operatorname{ord}_{\phi_{\tau_{0}}(\tau)} t(\tau), & \text { if }\left[\tau_{0}\right]_{N} \text { is a pole of } t^{*}\end{cases}
$$

Hence, we obtain Proposition 11.5.

${ }^{8}$ I.e., $\phi_{\tau_{0}}\left(\tau_{0}\right)=0$. 
Corollary 11.6. Let $t \in M^{\infty}(N)$ with $n:=\operatorname{pord} t \geq 1$ and $\left(1, b_{1}, \ldots, b_{n-1}\right)$ with $b_{j} \in M^{\infty}(N)$ be an integral basis for $M^{\infty}(N)$ over $\mathbb{C}[t]$. Then

$$
\operatorname{deg}_{x} D_{t}\left(1, b_{1}, \ldots, b_{n-1}\right)(x)=\sum_{\substack{x_{0} \in X_{0}(N), x_{0} \neq[\infty]_{N}}}\left(-1+\text { mult }_{x_{0}}\left(t^{*}\right)\right) .
$$

Next, recall from Sect. 16 the definition of $\operatorname{Deg}(f)$, the degree of a meromorphic function $f$ on a compact Riemann surface $X$ :

$$
\operatorname{Deg}(f):=\sum_{x \in f^{-1}(v)} \operatorname{mult}_{x}(f) \text { where } v \text { is any element in } \hat{\mathbb{C}} \text {. }
$$

Choosing $v:=\infty$, we have $\operatorname{Deg}\left(t^{*}\right)=n$. Let

$$
g(X):=\text { genus of a compact Riemann surface } X \text {. }
$$

Recall the Riemann-Hurwitz formula $[11 \text {, Thmorem 4.16 }]^{9}$ for a non-constant holomorphic map $F: X \rightarrow Y$ between compact Riemann surfaces:

$$
2 g(X)-2=\operatorname{Deg}(F)(2 g(Y)-2)+\sum_{x \in X}\left(\operatorname{mult}_{x}(F)-1\right) .
$$

Now, we apply this to our setting where $X:=X_{0}(N)$ and $F:=t^{*}: X_{0}(N) \rightarrow$ $\hat{\mathbb{C}}$. Owing to $g(\hat{\mathbb{C}})=0$, together with $(11.9)$ and Corollary 11.6, this gives

$$
\begin{aligned}
2 g\left(X_{0}(N)\right)-2 & =-2 n+\sum_{x \in X_{0}(N)}\left(\operatorname{mult}_{x}(F)-1\right) \\
& =-2 n+\sum_{\substack{x_{0} \in X_{0}(N), x_{0} \neq[\infty]_{N}}}\left(\operatorname{mult}_{x_{0}}\left(t^{*}\right)-1\right)+\text { pord } t-1 \\
& =-n-1+\operatorname{deg}_{x} D_{t}\left(1, b_{1}, \ldots, b_{n-1}\right)(x) .
\end{aligned}
$$

We summarize in

Corollary 11.7. Let $t \in M^{\infty}(N)$ with $n:=\operatorname{pord} t \geq 1$ and $\left(1, b_{1}, \ldots, b_{n-1}\right)$ with $b_{j} \in M^{\infty}(N)$ be an integral basis for $M^{\infty}(N)$ over $\mathbb{C}[t]$. Then

$$
\operatorname{deg}_{x} D_{t}\left(1, b_{1}, \ldots, b_{n-1}\right)(x)=2 g\left(X_{0}(N)\right)+n-1 .
$$

\section{Proof of the Weierstra $\beta$ Gap Theorem}

In this section, we prove the gap theorem for modular functions in $M^{\infty}(N)$.

Definition 12.1. (Gaps in modular function algebras) Let $M$ be a subalgebra of $M^{\infty}(N)$, the modular functions for $\Gamma_{0}(N)$ which are holomorphic in $\mathbb{H}$ and with a pole at $\infty$. A positive integer $n$ is called a gap in $M$ if there is no $f \in M$ with pord $f=n$. We also define the gap number $g_{M}$ as the total number of gaps in $M$, that is,

$$
g_{M}:=\#\left\{n \in \mathbb{Z}_{>0}: n \text { is a gap of } M\right\} .
$$

In this section, we prove the gap theorem in the following version.

\footnotetext{
${ }^{9}$ Actually the special case we need, $Y=\hat{\mathbb{C}}$, was given by Riemann; e.g., [2].
} 
Theorem 12.2. (Weierstraß gap theorem for $\left.X_{0}(N)\right)$ Let $g:=g\left(X_{0}(N)\right)$ be the genus of $X_{0}(N)$. If $g \geq 1$, then $M^{\infty}(N)$ has exactly $g$ gaps $n_{j}$ with

$$
1=n_{1}<\cdots<n_{g} \leq 2 g-1 .
$$

If $g=0$, then $M^{\infty}(N)$ has no gaps i.e., there exists an $h \in M^{\infty}(N)$, such that pord $h=1 .{ }^{10}$

To prepare for the proof, we determine the gap number $g_{\mathbb{C}[t, f]}$, where $t, f \in M^{\infty}(N)$ with $n:=\operatorname{pord} t \geq 2, l:=\operatorname{pord} f \geq 2$, and $\operatorname{gcd}(l, n)=1$. To construct such functions with relatively prime pole orders is straightforward; see, for instance, Example 2.3. By Proposition 3.5, we know that

$$
\mathbb{C}[t, f]=\left\langle 1, f, f^{2}, \ldots, f^{n-1}\right\rangle_{\mathbb{C}[t]},
$$

where $\left(1, f, f^{2}, \ldots, f^{n-1}\right)$ is an order-complete module basis. Hence, there are $l_{j} \in \mathbb{Z}_{\geq 0}, j=1,2, \ldots, n-1$, such that

$$
\begin{aligned}
\left\{\operatorname{pord} f, \operatorname{pord} f^{2}, \ldots, \text { pord } f^{n-1}\right\} & =\{l, 2 l, \ldots,(n-1) l\} \\
& =\left\{l_{1} n+1, l_{2} n+2, \ldots, l_{n-1} n+n-1\right\} .
\end{aligned}
$$

Thus, inspecting each of the residue classes modulo $n$ for $j \in\{1, \ldots, n-1\}$ makes clear that one cannot find any function of pole order

$$
j, n+j, \ldots,\left(l_{j}-1\right) n+j \text { if } l_{j}>0
$$

in $\mathbb{C}[t, f]$. Hence, for fixed $j, l_{j}$ pole orders are missing; summing $j$ from 1 to $n-1$ gives the total number of missing pole orders of functions in $\mathbb{C}[t, f]$ :

$$
\begin{aligned}
l_{1}+l_{2}+\cdots+l_{n-1} & =\left\lfloor\frac{l_{1} n+1}{n}\right\rfloor+\left\lfloor\frac{l_{2} n+2}{n}\right\rfloor+\cdots+\left\lfloor\frac{l_{n-1} n+n-1}{n}\right\rfloor \\
& =\sum_{j=1}^{n-1}\left\lfloor\frac{j l}{n}\right\rfloor=\frac{(l-1)(n-1)}{2},
\end{aligned}
$$

where the last equality is by $[8,(3.32)]$. We summarize in

Lemma 12.3. Let $t, f \in M^{\infty}(N)$ with $n:=\operatorname{pord} t \geq 1, l:=\operatorname{pord} f \geq 1$, and $\operatorname{gcd}(l, n)=1$. Then, the total number of missing pole orders of functions in $\mathbb{C}[t, f]$ is

$$
\frac{(l-1)(n-1)}{2} .
$$

Proof. If $n=1$ or $\ell=1$ then $\mathbb{C}[t, f]=M^{\infty}(N)$; i.e., there is no gap. The case both $n$ and $\ell$ greater or equal to 2 was treated above.

Proof of Theorem 12.2.. Recalling Definition 4.5, each pole-orderreduction step associated to some $\alpha \in \mathbb{C}$,

$$
\left(1, \ldots, \beta_{k-1}, \beta_{k}, \beta_{k+1}, \ldots\right) \rightarrow\left(1, \ldots, \beta_{k-1}, h_{\alpha}, \beta_{k+1}, \ldots\right)
$$

between order-complete bases

(i) by Corollary 4.4 (4.4) reduces the total number of gaps by exactly one.

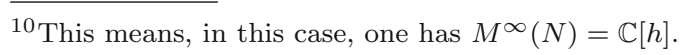


(ii) by Proposition 8.1 (8.3) reduces the degree of the order-reduction polynomial by exactly two.

The gap theorem now will be proved by successively applying pole-orderreduction steps to the order-complete basis $\left(1, f, \ldots, f^{n-1}\right)$ of $\mathbb{C}[t, f]$, where $t$ and $f$ are chosen from $M^{\infty}(N)$, such that $n:=\operatorname{pord} t \geq 2, l:=$ pord $f \geq 2$, and $\operatorname{gcd}(l, n)=1$. Such a pair $(t, f)$ can be easily constructed, see, for instance, Example 2.3.

Suppose that after $r$ reduction steps, we arrive at the integral basis $\left(1, b_{1}, \ldots, b_{n-1}\right)$ of $M^{\infty}(N)$. Defining

$$
d_{f}:=\operatorname{deg}_{x} D_{t}(f)(x) \text { and } d_{b}:=\operatorname{deg}_{x} D_{t}\left(1, b_{1}, \ldots, b_{n-1}\right)(x),
$$

the reduction observation (ii) made above gives

$$
d_{b}=d_{f}-2 r,
$$

furthermore, reduction observation (i) implies for the gap numbers:

$$
g_{M^{\infty}(N)}=g_{\mathbb{C}[t, f]}-r .
$$

Combining (12.2) and (12.3) gives the desired Weierstraß estimate for the total number of gaps in $M^{\infty}(N)$ in terms of the genus $g$ :

$$
\begin{aligned}
g_{M^{\infty}(N)} & =g_{\mathbb{C}[t, f]}-\frac{1}{2}\left(d_{f}-d_{b}\right) \quad(\text { by }(12.2),(12.3)) \\
& =\frac{1}{2}\left((n-1)(l-1)-d_{f}+d_{b}\right) \quad(\text { by Lemma 12.3) } \\
& =\frac{1}{2}\left(-(n-1)+d_{b}\right) \quad(\text { by }(7.1)) \\
& =\frac{1}{2}\left(-(n-1)+n-1+2 g\left(X_{0}(N)\right)\right) \quad(\text { by }(11.11)) \\
& =g\left(X_{0}(N)\right)=g .
\end{aligned}
$$

Hence, we proved that $M^{\infty}(N)$ has exactly $g$ gaps. If $g=0$ there are no gaps; i.e., in this case, after relabelling indices,

$$
M^{\infty}(N)=\left\langle 1, b_{1}, \ldots, b_{n-1}\right\rangle_{\mathbb{C}[t]} \text { with } \operatorname{pord} b_{j}=j, j=1, \ldots, n-1 .
$$

Hence, $M^{\infty}(N)=\mathbb{C}[h]$ for $h:=b_{1}$.

To prove the remaining part of the gap theorem, namely, the bound (12.1) for the gaps $\left\{n_{1}=1, n_{2}, \ldots, n_{g}\right\}$ where $g \geq 1$, we will use a general combinatorial argument. Notice that $n_{1}=1$, because otherwise there would be no gap, which, as we proved, is only possible if $g=0$.

To prepare for the combinatorial argument, recall that after choosing $t$ and $f$ from $M^{\infty}(N)$ as above, by applying pole-order-reduction steps, we arrived, after relabelling indices, at an integral basis $\left(1, b_{1}, \ldots, b_{n-1}\right)$ for $M^{\infty}(N)$ where pord $b_{j} \equiv j(\bmod n), j=1, \ldots, n-1$. Defining

$$
r_{1}:=\operatorname{pord} b_{1}, \ldots, r_{n-1}:=\operatorname{pord} b_{n-1},
$$

this basis gives rise to the additive submonoid

$$
S:=\left(0+n \mathbb{Z}_{\geq 0}\right) \cup\left(r_{1}+n \mathbb{Z}_{\geq 0}\right) \cup \cdots \cup\left(r_{n-1}+n \mathbb{Z}_{\geq 0}\right)
$$


of $\left(\mathbb{Z}_{\geq 0},+\right)$ which describes the gap set of $M^{\infty}(N)$ :

$$
\mathbb{Z}_{\geq 0} \backslash S=\left\{n_{1}=1, n_{2}, \ldots, n_{g}\right\} .
$$

Let $m+1$ be the smallest non-gap of $M^{\infty}(N) ; m \geq 1$ owing to $n_{1}=1$.

To prove the desired bound (12.1) for the gap sizes $n_{j}$, we change the representation of the monoid $S$ with respect to $m+1$. Namely, it is easy to see that there exist positive integers $s_{1}, \ldots, s_{m} \in \mathbb{Z}_{>0}$, such that $s_{j} \equiv j$ $(\bmod m+1)$ for all $j \in\{1, \ldots, m\}$ and

$$
S=\left(0+(m+1) \mathbb{Z}_{\geq 0}\right) \cup\left(s_{1}+(m+1) \mathbb{Z}_{\geq 0}\right) \cup \cdots \cup\left(s_{m}+(m+1) \mathbb{Z}_{\geq 0}\right) .
$$

In Sect. 13, we denote the number of gaps in a monoid $S$ by $\gamma(S)$. Hence, in the given context, $g=\gamma(S)$. Recall that $m+1$ is chosen to be the smallest nongap of $M^{\infty}(N)$. Therefore, we choose a monoid representation with respect to $m+1$. Concretely, in this case, there are $k_{j} \in \mathbb{Z}_{>0}$, such that

$$
s_{j}=j+(m+1) k_{j} \text { for } j=1, \ldots, m .
$$

Now, Lemma 13.1 implies

$$
2 \gamma(S)-1 \geq j+(m+1)\left(k_{j}-1\right), j=1, \ldots, m .
$$

Since $j+(m+1)\left(k_{j}-1\right)$ are the largest non-gaps in each residue class modulo $m+1$, this proves the bound given in (12.1), and the proof of the Weierstraß gap Theorem 12.2 is completed.

\section{A Gap Property of Monoids}

Let $m \in \mathbb{Z}_{>0}$ and $s_{1}, \ldots, s_{m} \in \mathbb{Z}_{>0}$, such that $s_{j} \equiv j(\bmod m+1)$ for all $j \in\{1, \ldots, m\}$. We consider the additive submonoid:

$$
S:=\left(0+(m+1) \mathbb{Z}_{\geq 0}\right) \cup\left(s_{1}+(m+1) \mathbb{Z}_{\geq 0}\right) \cup \cdots \cup\left(s_{m}+(m+1) \mathbb{Z}_{\geq 0}\right)
$$

of $\left(\mathbb{Z}_{\geq 0},+\right)$. A positive integer $\ell \notin S$ is called a gap of $S .{ }^{11}$ Let $\gamma(S)$ be the total number of gaps of $S$. Relating to our proof setting in Sect. 12, we choose this representation of $S$ under the assumption that $m+1$ is the smallest non-gap of $S$. By the definition of $S$, there are positive integers $k_{j}$, such that

$$
s_{j}=j+(m+1) k_{j} \quad \text { for } j=1, \ldots, m .
$$

An easy count gives

$$
\gamma(S)=k_{1}+\cdots+k_{m}
$$

Lemma 13.1 (Monoid gap lemma). Under these assumptions, one has for all $j=1, \ldots, m$ :

$$
2 \gamma(S)-1 \geq j+(m+1)\left(k_{j}-1\right)
$$

In other words, the largest possible gap is bounded by $2 \gamma(S)-1$. Before proving this statement, we prove two elementary observations.

\footnotetext{
${ }^{11}$ The largest gap is called the Frobenius number of $S$.
} 
Lemma 13.2. If $i$ and $\ell$ in $\mathbb{Z}_{>0}$ are such that $i+\ell=j$ for $j \in\{1, \ldots, m\}$, then

$$
k_{i}+k_{\ell} \geq k_{j}
$$

Proof.

$$
\begin{aligned}
s_{i}+s_{\ell} & =i+(m+1) k_{i}+\ell+(m+1) k_{\ell} \\
& =j+(m+1)\left(k_{i}+k_{\ell}\right) \geq j+(m+1) k_{j} .
\end{aligned}
$$

The inequality is by $s_{i}+s_{\ell} \in S$ with $s_{i}+s_{\ell} \equiv j(\bmod m+1)$, and $s_{j} \in S$ is minimal with this property.

Lemma 13.3. If $i$ and $\ell$ in $\mathbb{Z}_{>0}$ are such that $i+\ell=j+m+1$ for $j \in\{1, \ldots, m\}$, then $k_{i}+k_{\ell}+1 \geq k_{j}$.

Proof.

$$
s_{i}+s_{\ell}=i+(m+1) k_{i}+\ell+(m+1) k_{\ell}=j+(m+1)\left(k_{i}+k_{\ell}+1\right) \geq s_{j} .
$$

The inequality is by $s_{i}+s_{\ell} \in S$ with $s_{i}+s_{\ell} \equiv j(\bmod m+1)$, and $s_{j}=$ $j+(m+1) k_{j} \in S$ is minimal with this property.

Proof of Lemma 13.1. By (13.1), the statement to prove is equivalent to

$$
2\left(k_{1}+\cdots+k_{m}\right) \geq j+(m+1) k_{j}-m
$$

By Lemma 13.2,

$$
k_{j} \leq k_{1}+k_{j-1}, k_{j} \leq k_{2}+k_{j-2}, \ldots, k_{j} \leq k_{j-1}+k_{1} .
$$

Summing the left and right sides, respectively; of these, $j-1$ inequalities gives

$$
(j-1) k_{j} \leq 2\left(k_{1}+\cdots+k_{j-1}\right) .
$$

By Lemma 13.3,

$$
k_{j} \leq k_{j+1}+k_{m+1-1}+1, k_{j} \leq k_{j+2}+k_{m+1-2}+1, \ldots, k_{j} \leq k_{m}+k_{j+1}+1 .
$$

Summing the left and right sides, respectively; of these, $m-j$ inequalities gives

$$
(m-j) k_{j} \leq 2\left(k_{j+1}+\cdots+k_{m}\right)+m-j .
$$

Combining the two inequalities, we obtain that

$$
(m-1) k_{j} \leq 2\left(k_{1}+\cdots+k_{m}\right)-2 k_{j}+m-j,
$$

which is $(13.3)$. 


\section{Functions with Separation Property}

The setting which we use throughout this section is: $t \in M^{\infty}(N)$ with $n:=$ pord $t \geq 2$ and $\left(1, b_{1}, \ldots, b_{n-1}\right)$ with $b_{j} \in M^{\infty}(N)$ is an integral basis for $M^{\infty}(N)$ over $\mathbb{C}[t]$. Because of pord $t=n$, for any fixed $\alpha \in \mathbb{C}$, we have that ${ }^{12}$

$t^{*}(x)=\alpha$ has $\ell \leq n$ pairwise distinct solutions $x_{1}=\left[\tau_{1}\right]_{N}, \ldots, x_{\ell}=\left[\tau_{\ell}\right]_{N}$, with multiplicities $k_{1}, \ldots, k_{\ell}$, respectively. (I.e., $k_{1}+\cdots+k_{\ell}=n$.) (14.1)

We note that, as above, owing to $t \in M^{\infty}(N), x_{j}=\left[\tau_{j}\right]_{N} \in \Gamma_{0}(N)$ with $\tau_{j} \in \mathbb{H} \cup \mathbb{Q}$ are such that $\left[\tau_{j}\right]_{N} \neq[\infty]_{N}$.

In Definition 9.4, we defined the separation property of $f$ for $\left(t, v_{0}\right)$ with $v_{0}=\alpha$ as in (14.1). At various places, we required $f$ to have this property, for instance, in Proposition 11.5. In this section, we prove the existence of such $f$. In addition, here, we have to use the charts as in (9.1), (9.2), and (9.3).

Lemma 14.1. Given the setting of this section with $\ell \geq 2$, let $\tau, \tau^{\prime} \in\left\{\tau_{1}, \ldots, \tau_{\ell}\right\}$ be such that $\tau \neq \tau^{\prime}$. Then

$$
b_{i}(\tau) \neq b_{i}\left(\tau^{\prime}\right) \text { for some } i \in\{1, \ldots, n-1\} .
$$

Proof. We are free to relabel the indices of the preimages of $\alpha$. Hence, it is sufficient to prove the statement for $\tau_{1}:=\tau$ and $\tau_{2}:=\tau^{\prime}$. Suppose

$$
b_{j}\left(\tau_{1}\right)=b_{j}\left(\tau_{2}\right) \text { for all } j \in\{1, \ldots, n-1\} .
$$

As in (9.7), for $j=1, \ldots, \ell$ and suitable neighborhoods $U_{j}$, one has local expansions for $\tau \in U_{j}$ :

$$
t(\tau)-\alpha=a_{j, 0} \phi_{\tau_{j}}(\tau)^{k_{j}}+a_{j, 1} \phi_{\tau_{j}}(\tau)^{k_{j}+1}+\cdots \text { with } a_{j, 0} \neq 0 .
$$

Moreover, as in (9.10), for $j=1, \ldots, \ell$, we can assume that the neighborhoods $U_{j}$ are chosen, such that the following expansions exist for all $\tau \in U_{j}$ :

$$
\begin{gathered}
b_{1}(\tau)=b_{1}\left(\tau_{j}\right)+\sum_{l=1}^{\infty} d_{l}^{(1, j)} \phi_{\tau_{j}}(\tau)^{l}, \\
\vdots \\
b_{n-1}(\tau)=b_{n-1}\left(\tau_{j}\right)+\sum_{l=1}^{\infty} d_{l}^{(n-1, j)} \phi_{\tau_{j}}(\tau)^{l} .
\end{gathered}
$$

Taking $a_{j} \in \mathbb{C}$ the quotient

$$
g:=\frac{a_{0}+a_{1} b_{1}+\cdots+a_{n-1} b_{n-1}}{t-\alpha}
$$

defines a modular function $g \in M(N)$. Now, $g \in M^{\infty}(N)$ if and only if all the zeros $\tau_{j}$ of $t\left(\tau_{j}\right)-\alpha=0$ cancel out. Indeed, assuming $(\star)$, one can determine $a_{j} \in \mathbb{C}$, not all zero, such that this cancellation happens. Namely, using the

\footnotetext{
${ }^{12}$ Recall that $t^{*}\left([\tau]_{n}\right)=t(\tau)$.
} 
local expansions the cancellation condition translates into a system of linear equations, where $j$ runs form 1 to $\ell:^{13}$

$$
\begin{gathered}
a_{0}+a_{1} b_{1}\left(\tau_{j}\right)+\cdots+a_{n-1} b_{n-1}\left(\tau_{j}\right)=0 \\
a_{1} d_{1}^{(1, j)}+\cdots+a_{n-1} d_{1}^{(n-1, j)}=0 \\
\vdots \\
a_{1} d_{k_{j}-1}^{(1, j)}+\cdots+a_{n-1} d_{k_{j}-1}^{(n-1, j)}=0
\end{gathered}
$$

This gives in total $k_{1}+\cdots+k_{\ell}=n$ equations. However, owing to $(\star)$, two of these equations are the same. This means, we are left with $n-1$ equations in $n$ unknowns $a_{0}, \ldots, a_{n-1}$. This implies that there exists a solution to the system with the $a_{j}$ not all 0 . This produces a contradiction: since $g \in M^{\infty}(N)$, there are polynomials $p_{j}(x) \in \mathbb{C}[x]$, such that

$$
g=p_{0}(t)+p_{1}(t) b_{1}+\cdots+p_{n-1}(t) b_{n-1} .
$$

Combining this with (14.2), the uniqueness of the basis representation gives

$$
a_{0}=(t-\alpha) p_{0}(t), \ldots, a_{n-1}=(t-\alpha) p_{n-1}(t) .
$$

Consequently, all $p_{j}(x)$ and all $a_{j}$ must be zero. Hence, $(\star)$ leads to a contradiction and the lemma is proved.

Lemma 14.2. Given the setting of this section with $\ell \geq 2$, let

$$
S_{m}:=\left\{\left[\tau_{i_{1}}\right]_{N}, \ldots,\left[\tau_{i_{m}}\right]_{N}\right\}
$$

be a subset of $m \in\{2, \ldots, \ell\}$ pairwise distinct preimages of $\alpha$. Then, there exist $\alpha_{i} \in \mathbb{C}$, such that for $f=\alpha_{1} b_{1}+\cdots+\alpha_{n-1} b_{n-1}$ :

$$
f\left(\tau_{i_{1}}\right), \ldots, f\left(\tau_{i_{m}}\right) \text { are pairwise distinct. }
$$

Proof. We proceed by induction on $m$. If $m=2$, then by the previous lemma, there exists an $i \in\{1, \ldots, n-1\}$, such that $b_{i}\left(\tau_{i_{1}}\right) \neq b_{i}\left(\tau_{i_{2}}\right)$, and we choose $f:=b_{i}$. Suppose $m \geq 2$. Because of index relabeling, we can choose $S_{m}:=$ $\left\{\tau_{1}, \ldots, \tau_{m}\right\}$, and the induction hypothesis gives an $F \in M^{\infty}(N)$ as a $\mathbb{C}$-linear combination of $b_{j}$, such that the values $F\left(\tau_{1}\right), \ldots, F\left(\tau_{m}\right)$ are pairwise distinct. If $F\left(\tau_{m+1}\right) \neq F\left(\tau_{i}\right)$ for all $i=1, \ldots, m$, the induction step is done. Otherwise, $F\left(\tau_{m+1}\right)=F\left(\tau_{r}\right)$ for some $r \in\{1, \ldots, m\}$. By the previous lemma, there is some $k \in\{1, \ldots, n-1\}$, such that $b_{k}\left(\tau_{m+1}\right) \neq b_{k}\left(\tau_{r}\right)$, and we can choose a non-zero $c \in \mathbb{C}$, such that

$$
c \neq \frac{F\left(\tau_{j}\right)-F\left(\tau_{i}\right)}{b_{k}\left(\tau_{i}\right)-b_{k}\left(\tau_{j}\right)} \text { for all } 1 \leq i<j \leq m+1 \text { with } b_{k}\left(\tau_{i}\right) \neq b_{k}\left(\tau_{j}\right) .
$$

By inspection, one verifies for $F_{r}:=F+c b_{k}$ that $F_{r}\left(\tau_{m+1}\right) \neq F_{r}\left(\tau_{r}\right)$ and also that the values

$$
F_{r}\left(\tau_{1}\right), \ldots, F_{r}\left(\tau_{m}\right) \text { are pairwise distinct. }
$$

\footnotetext{
${ }^{13}$ Notice that the charts $\phi_{\tau_{j}}(\tau)$ are centered at 0 ; i.e., $\phi_{\tau_{j}}\left(\tau_{j}\right)=0$. Consequently, for fixed $j$, the numerator in (14.2) has to be of the form: $\phi_{\tau_{j}}(\tau)^{k_{j}}\left(c_{0}+c_{1} \phi_{\tau_{j}}(\tau)+\cdots\right)$.
} 
Suppose $F_{r}\left(\tau_{m+1}\right)=F_{r}\left(\tau_{s}\right)$ for some $s \in\{1, \ldots, m\} \backslash\{r\}$. If there is no such $s$, we are done with $f:=F_{r}$. Otherwise, by the previous lemma, there is some $l \in\{1, \ldots, n-1\}$, such that $b_{l}\left(\tau_{m+1}\right) \neq b_{l}\left(\tau_{s}\right)$, and we can choose a non-zero $d \in \mathbb{C}$, such that

$$
d \neq \frac{F_{r}\left(\tau_{j}\right)-F_{r}\left(\tau_{i}\right)}{b_{l}\left(\tau_{i}\right)-b_{l}\left(\tau_{j}\right)} \text { for all } 1 \leq i<j \leq m+1 \text { with } b_{l}\left(\tau_{i}\right) \neq b_{l}\left(\tau_{j}\right) .
$$

Now, we set $F_{r, s}:=F_{r}+d b_{l}$, and see that,

$$
F_{r, s}\left(\tau_{m+1}\right) \neq F_{r, s}\left(\tau_{r}\right) \text { and } F_{r, s}\left(\tau_{m+1}\right) \neq F_{r, s}\left(\tau_{s}\right)
$$

together with pairwise distinct values $F_{r, s}\left(\tau_{1}\right), \ldots, F_{r, s}\left(\tau_{m}\right)$. Iterating this argument exhausts all possibilities and the induction step is proved.

Under the assumptions as in (14.1), to have the separation property for $(t, \alpha), f$ additionally has to satisfy the conditions (9.13) which rewritten as order conditions are

$$
\operatorname{ord}_{\phi_{\tau_{j}}(\tau)}(f(\tau)-\alpha)=1 \text { for all } j=1, \ldots, \ell .
$$

Remark 14.3. The order in (14.4) has to be interpreted in view of (9.10) and in the sense of the $\phi$-order defined in Definition 15.3. This deviates slightly from the standard notation used in the theory of the Riemann surfaces, where (14.4) would be stated in the format:

$$
\operatorname{ord}_{\tau_{j}}(f-\alpha)=1 \text { for all } j=1, \ldots, \ell \text {. }
$$

This notation suppresses the explicit mentioning of the chart. We also use this notation, for instance, in cases like (2.4), where the chart is clear from the context, or in Lemma 16.1 when citing from Riemann surface theory.

Lemma 14.4. Given the setting of this section with $\ell \geq 1$, assume that $k_{i}=$ $\operatorname{ord}_{\phi_{\tau_{i}}(\tau)}(t(\tau)-\alpha)>1$ for some $i \in\{1, \ldots, n-1\}$. Then

$$
\operatorname{ord}_{\phi_{\tau_{i}}(\tau)}\left(b_{j}(\tau)-b_{j}\left(\tau_{i}\right)\right)=1 \text { for some } j \in\{1, \ldots, n-1\} \text {. }
$$

Proof. Let us assume that

$$
\operatorname{ord}_{\phi_{\tau_{i}}(\tau)}\left(b_{k}(\tau)-b_{k}\left(\tau_{i}\right)\right)>1 \text { for all } k \in\{1, \ldots, n-1\} .
$$

As in (9.7), for $j=1, \ldots, \ell$ and suitable neighborhoods $U_{j}$, one has local expansions for $\tau \in U_{j}$ :

$$
t(\tau)-\alpha=a_{j, 0} \phi_{\tau_{j}}(\tau)^{k_{j}}+a_{j, 1} \phi_{\tau_{j}}(\tau)^{k_{j}+1}+\cdots \text { with } a_{j, 0} \neq 0 .
$$

Now, we proceed with the proof exactly as above. Namely, as in (9.10), for $j=1, \ldots, \ell$, we can assume that the neighborhoods $U_{j}$ are chosen such that the following expansions exist for all $\tau \in U_{j}$ and $k \in\{1, \ldots, n-1\}$ :

$$
b_{k}(\tau)=b_{k}\left(\tau_{j}\right)+\sum_{l=1}^{\infty} d_{l}^{(k, j)} \phi_{\tau_{j}}(\tau)^{l}
$$


Now, we apply the same strategy as in the proof of Lemma 14.1 and determine $a_{j} \in \mathbb{C}$, not all zero, such that

$$
g:=\frac{a_{0}+a_{1} b_{1}+\cdots+a_{n-1} b_{n-1}}{t-\alpha} \in M^{\infty}(N) .
$$

This leads us to consider the same system of $k_{1}+\cdots+k_{\ell}=n$ linear equations. This time, owing to $(\star \star)$, we have $d_{1}^{(k, i)}=0$ for all $k=1, \ldots, n-1$, and the equation containing the $d_{1}^{(k, i)}$ is always satisfied and can be removed. This means, we are left with $n-1$ equations in $n$ unknowns $a_{0}, \ldots, a_{n-1} \cdot{ }^{14}$ This implies that there exists a solution to the system with the $a_{j}$ not all 0 , which produces a contradiction as in the proof of Lemma 14.2.

Lemma 14.5. Again, we assume the setting of this section with $\ell \geq 1$. Then, there exist $\alpha_{i} \in \mathbb{C}$, such that for $f:=\alpha_{0} t+\alpha_{1} b_{1}+\cdots+\alpha_{n-1} b_{n-1}$ :

$$
\begin{aligned}
& f\left(\tau_{1}\right), \ldots, f\left(\tau_{\ell}\right) \text { are pairwise distinct, and } \\
& \operatorname{ord}_{\phi_{\tau_{j}}(\tau)}\left(f(\tau)-f\left(\tau_{j}\right)\right)=1, \quad j=1, \ldots, \ell
\end{aligned}
$$

Proof. For the proof, it is convenient to introduce an auxiliary function:

$$
g_{i}(\tau):=\left\{\begin{array}{ll}
t(\tau), & \text { if } \operatorname{ord}_{\phi_{\tau_{i}}(\tau)}(t(\tau)-\alpha)=1, \\
b_{j}(\tau), & \text { if } \operatorname{ord}_{\phi_{\tau_{i}}(\tau)}(t(\tau)-\alpha)>1,
\end{array} \quad i=1, \ldots, \ell,\right.
$$

where $j$ is chosen according to Lemma 14.4, namely, such that ord $\operatorname{dit}_{\tau_{i}}(\tau)\left(b_{j}(\tau)-\right.$ $\left.b_{j}\left(\tau_{i}\right)\right)=1$. We will show: given $F=a_{0} t+a_{1} b_{1}+\cdots+a_{n-1} b_{n-1}$ with $a_{j} \in \mathbb{C}$, such that

$$
\begin{array}{r}
F\left(\tau_{1}\right), \ldots, F\left(\tau_{\ell}\right) \text { are pairwise distinct, and } \\
\operatorname{ord}_{\phi_{\tau_{j}}(\tau)}\left(F(\tau)-F\left(\tau_{j}\right)\right)=1 \text { for } j=1, \ldots, k,
\end{array}
$$

then there is an $f=\alpha_{0} t+\alpha_{1} b_{1}+\cdots+\alpha_{n-1} b_{n-1}$ with $\alpha_{j} \in \mathbb{C}$ which satisfies (14.6) and

$$
\operatorname{ord}_{\phi_{\tau_{j}}(\tau)}\left(f(\tau)-f\left(\tau_{j}\right)\right)=1 \text { for } j=1, \ldots, k+1 .
$$

In other words, we prove Lemma 14.5 by induction on $k$.

The base case $k=1$ corresponds to the induction step from $k=0$ to $k=1$. The existence of $F$ such that (14.8) holds is by Lemma 14.2. If, in addition,

$$
\operatorname{ord}_{\phi_{\tau_{1}}(\tau)}\left(F(\tau)-F\left(\tau_{1}\right)\right)=1
$$

we take $f:=F$, and the base case $k=1$ is done. If $\operatorname{ord}_{\phi_{\tau_{1}}(\tau)}\left(F(\tau)-F\left(\tau_{1}\right)\right)>1$, define

$$
f:=F+c g_{1} \text { with } c \in \mathbb{C} \backslash\{0\} \text { such that } c \neq \frac{F\left(\tau_{j}\right)-F\left(\tau_{i}\right)}{g_{1}\left(\tau_{i}\right)-g_{1}\left(\tau_{j}\right)},
$$

\footnotetext{
${ }^{14}$ Notice that for this argument to work, we invoke $k_{i}>1$.
} 
where the quotient is taken for all $i, j \in\{1, \ldots, \ell\}$ for which the denominator is non-zero. Now, it is a straightforward verification that for this $f$ the condition (14.6) holds and also that

$$
\operatorname{ord}_{\phi_{\tau_{1}}(\tau)}\left(f(\tau)-f\left(\tau_{1}\right)\right)=1
$$

This settles the base case $k=1$.

For the induction step $k \rightarrow k+1$, we assume that we have $F$ of required form, such that (14.8) and (14.9) hold. If, in addition,

$$
\operatorname{ord}_{\phi_{\tau_{k+1}}(\tau)}\left(F(\tau)-F\left(\tau_{k+1}\right)\right)=1
$$

we are done. Otherwise, define

$$
f:=F+c g_{k+1} \text { with } c \in \mathbb{C} \backslash\{0\} \text { such that } c \neq \frac{F\left(\tau_{j}\right)-F\left(\tau_{i}\right)}{g_{k+1}\left(\tau_{i}\right)-g_{k+1}\left(\tau_{j}\right)},
$$

where the quotient is taken for all $i, j \in\{1, \ldots, \ell\}$ for which the denominator is non-zero. Now, we additionally require that

$$
c \neq \frac{a_{1, j}}{b_{1, j}} \text { for all } j \in\{1, \ldots, \ell\} \text { when } b_{1, j} \neq 0,
$$

for $a_{1, j}$ and $b_{1, j}$ coming from the expansions:

$$
F(\tau)=a_{0, j}+a_{1, j} \phi_{j}(\tau)+\cdots \text { and } g_{k+1}(\tau):=b_{0, j}+b_{1, j} \phi_{j}(\tau)+\cdots .
$$

Again, it is straightforward to verify that for such a choice $f$ has the properties (14.6) and (14.10). The extra requirement (14.11) is needed to guarantee the first $k$ instances of the latter condition. This completes the proof of the induction step and also the proof of Lemma 14.5.

Summarizing, using an integral basis $\left(1, b_{1}, \ldots, b_{n-1}\right)$ for $M^{\infty}(N)$ over $\mathbb{C}[t]$, we constructed an $f$ which proves

Corollary 14.6. For every $\alpha \in \mathbb{C}$, there is an $f \in M^{\infty}(N)$, such that $f$ has the separation property for $(t, \alpha)$.

\section{Appendix: Modular Functions-Basic Notions}

To make this article as much self-contained as possible, in this section, we recall most of the facts we need about modular functions.

The modular group $\mathrm{SL}_{2}(\mathbb{Z})=\left\{\left(\begin{array}{ll}a & b \\ c & d\end{array}\right) \in \mathbb{Z}^{2 \times 2}: a d-b c=1\right\}$ acts on the upper half $\mathbb{H}$ of the complex plane by $\left(\begin{array}{ll}a & b \\ c & d\end{array}\right) \tau:=\frac{a \tau+b}{c \tau+d}$; this action is inherited by the congruence subgroups:

$$
\Gamma_{0}(N):=\left\{\left(\begin{array}{ll}
a & b \\
c & d
\end{array}\right) \in \mathrm{SL}_{2}(\mathbb{Z}): N \mid c\right\}
$$

where throughout this paper, $N$ is a fixed positive integer. Note that $\Gamma_{0}(1)=$ $\mathrm{SL}_{2}(\mathbb{Z})$. These subgroups have a finite index in $\mathrm{SL}_{2}(\mathbb{Z})$ :

$$
\left[\mathrm{SL}_{2}(\mathbb{Z}): \Gamma_{0}(N)\right]=N \prod_{\text {prime } p \mid N}\left(1+\frac{1}{p}\right), \quad N \geq 2
$$

see the standard literature on modular forms like [1] or [5]. Particularly related to our context are [9] and [12]. 
The action of $\mathrm{SL}_{2}(\mathbb{Z})$ on $\mathbb{H}$ extends to an action on meromorphic functions $f: \mathbb{H} \rightarrow \hat{\mathbb{C}}:=\mathbb{C} \cup\{\infty\}$. A meromorphic function $f: \mathbb{H} \rightarrow \hat{\mathbb{C}}$ is called a meromorphic modular function for $\Gamma_{0}(N)$, if (i) for all $\left(\begin{array}{ll}a & b \\ c & d\end{array}\right) \in \Gamma_{0}(N)$ :

$$
f\left(\frac{a \tau+b}{c \tau+d}\right)=f(\tau), \quad \tau \in \mathbb{H},
$$

and (ii) for any $\gamma=\left(\begin{array}{ll}a & b \\ c & d\end{array}\right) \in \mathrm{SL}_{2}(\mathbb{Z})$, there exists an $M=M(\gamma) \in \mathbb{Z}$ together with a Fourier expansion:

$$
f(\gamma \tau)=f\left(\frac{a \tau+b}{c \tau+d}\right)=\sum_{n=-M}^{\infty} f_{n}(\gamma) q^{n / w_{N}(c)},
$$

where $q=q(\tau):=e^{2 \pi i \tau}$ and $w_{N}(c):=N / \operatorname{gcd}\left(c^{2}, N\right)$. By $M(N)$, we denote the set of meromorphic modular functions for $\Gamma_{0}(N)$.

By (ii) with $\left(\begin{array}{ll}a & b \\ c & d\end{array}\right)=\left(\begin{array}{ll}1 & 0 \\ 0 & 1\end{array}\right)$, any $f \in M(N)$ admits a Laurent series expansion in powers of $q$ with finite principal part, that is

$$
f(\tau)=\sum_{n=-M}^{\infty} f_{n} q^{n}
$$

Hence, in view of $\lim _{\operatorname{Im}(\tau) \rightarrow \infty} q(\tau)=0$, one can extend $f$ to $\mathbb{H} \cup\{\infty\}$ by defining $f(\infty):=\infty$, if $M>0$, and $f(\infty):=f_{0}$, otherwise. Subsequently, a Laurent expansion of $f$ as in (15.2) will be also called q-expansion of $f$ at infinity. ${ }^{15}$

Given $\gamma=\left(\begin{array}{ll}a & b \\ c & d\end{array}\right) \in \mathrm{SL}_{2}(\mathbb{Z})$ and $f \in M(N)$, consider the Laurent series expansion of $f(\gamma \tau)$ in powers of $q^{1 / w_{N}(c)}$,

$$
f(\gamma \tau)=\sum_{n=-M}^{\infty} g_{n} q^{n / w_{N}(c)} .
$$

In view of $\gamma \infty=\lim _{\operatorname{Im}(\tau) \rightarrow \infty} \gamma \tau=a / c$, we say that (15.3) is a $q$-expansion of $f$ at $a / c$. Understanding that $a / 0=\infty$, this also covers the definition of $q$-expansions at $\infty$. Concerning the uniqueness of such expansions, let $\gamma^{\prime} \in$ $\mathrm{SL}_{2}(\mathbb{Z})$ be such that $\gamma^{\prime} \infty=\gamma \infty=a / c$, then the $q$-expansion of $f\left(\gamma^{\prime} \tau\right)$ differs from that of $f(\gamma \tau)$ only by a root-of-unity factor in the coefficients, namely, we have then $\gamma^{\prime}=\gamma\left(\begin{array}{cc} \pm 1 & m \\ 0 & \pm 1\end{array}\right)$ for some $m \in \mathbb{Z}$, which implies

$$
f\left(\gamma^{\prime} \tau\right)=\sum_{n=-M}^{\infty} g_{n}\left(e^{ \pm 2 \pi i m / w_{N}(c)}\right)^{n} q^{n / w_{N}(c)} .
$$

As a consequence, one can extend $f$ from $\mathbb{H}$ to $\hat{\mathbb{H}}:=\mathbb{H} \cup\{\infty\} \cup \mathbb{Q}$ by defining $f(a / c):=\lim _{\operatorname{Im}(\tau) \rightarrow \infty} f(\gamma \tau)$, where $\gamma \in \mathrm{SL}_{2}(\mathbb{Z})$ is chosen, such that $\gamma \infty=$ $a / c$. Another consequence is that the $q$-expansions of $f$ at $\infty$ are uniquely determined owing to

$$
\gamma \infty=\infty \Leftrightarrow \gamma=\left(\begin{array}{cc} 
\pm 1 & m \\
0 & \pm 1
\end{array}\right) \text { and } w_{\ell}(0)=1 .
$$

\footnotetext{
${ }^{15}$ This expansion and also those for $f(\gamma \tau)$ are required to converge for all $\tau \in \mathbb{H}$ with $\operatorname{Im}(\tau)$ sufficiently large.
} 
Next, notice that the action of $\mathrm{SL}_{2}(\mathbb{Z})$, and thus of $\Gamma_{0}(N)$, extends in an obvious way to an action on $\hat{\mathbb{H}}$. The orbits of the $\Gamma_{0}(N)$ action are denoted by

$$
[\tau]_{N}:=\left\{\gamma \tau: \gamma \in \Gamma_{0}(N)\right\}, \quad \tau \in \hat{\mathbb{H}} .
$$

In cases where $N$ is clear from the context, one also writes $[\tau]$ instead of $[\tau]_{N}$. The set of all such orbits is denoted by

$$
X_{0}(N):=\left\{[\tau]_{N}: \tau \in \hat{\mathbb{H}}\right\} .
$$

The $\Gamma_{0}(N)$ action maps $\mathbb{Q} \cup\{\infty\}$ to itself, and owing to $(15.1)$, each $\Gamma_{0}(N)$ produces only finitely many orbits $[\tau]_{N}$ with $\tau \in \mathbb{Q} \cup\{\infty\}$; such orbits are called cusps of $X_{0}(N)$. One has, for example,

Lemma 15.1. For any prime $\ell$

(1) $X_{0}(\ell)$ has two cusps $:[\infty]_{\ell}$ and $[0]_{\ell}$;

(2) $X_{0}\left(\ell^{2}\right)$ has $\ell+1$ cusps $:[\infty]_{\ell^{2}},[0]_{\ell^{2}}$, and $[k / \ell]_{\ell^{2}}, k=1, \ldots, \ell-1$.

Proof. This fact can be found in many sources; a detailed description of how to construct a set of representatives for the cusps of $\Gamma_{0}(N)$, for instance, is given in [16, Lemma 5.3].

Suppose that the domain of $f \in M(N)$ is extended from $\mathbb{H}$ to $\hat{\mathbb{H}}$ as described above, i.e., $f: \mathbb{H} \rightarrow \hat{\mathbb{C}}$ is extended to $f: \hat{\mathbb{H}} \rightarrow \hat{\mathbb{C}}$, where we keep the same name for the extended function. Then, using this extension gives rise to a function $f^{*}: X_{0}(N) \rightarrow \hat{\mathbb{C}}$, which is defined as follows:

$$
f^{*}\left([\tau]_{N}\right):=f(\tau), \quad \tau \in \hat{\mathbb{H}} .
$$

The fact that $f^{*}$ is well-defined follows from our previous discussion. We say that $f^{*}$ is induced by $f$.

As described in detail in [5], $X_{0}(N)$ can be equipped with the structure of a compact Riemann surface. This analytic structure turns the induced functions $f^{*}$ into meromorphic functions on $X_{0}(N)$. The following classical lemma [11, Theorem 1.37], a Riemann surface version of Liouville's theorem, is crucial for zero recognition of modular functions.

Lemma 15.2. Let $X$ be a compact Riemann surface. Suppose that $g: X \rightarrow \mathbb{C}$ is a holomorphic function on all of $X$. Then, $g$ is a constant function.

Being meromorphic, modular functions form fields. A classic example is that $M(N)=\mathbb{C}(j(\tau), j(N \tau))$, e.g., [5, Proposition 7.5.1], where $j$ is the modular invariant (the Klein $j$ function). The subset

$$
M^{!}(N):=\left\{f \in M(N): f^{*} \text { has poles only at }[\tau]_{N} \text { with } \tau \in \mathbb{Q} \cup\{\infty\}\right\},
$$

which is important for our context and, obviously, is not a field but a $\mathbb{C}$ algebra. In this case, owing to the definition of induced functions $f^{*}$, all possible poles of $f^{*}$ can be spotted by checking whether $f^{*}([a / c])=f(a / c)=\infty$ for $a / c \in \mathbb{Q} \cup\{\infty\}$. Because of (15.1), $\mathbb{Q} \cup\{\infty\}$ splits only into a finite number of cusps:

$$
\mathbb{Q} \cup\{\infty\}=\left[a_{1} / c_{1}\right]_{N} \cup \cdots \cup\left[a_{k} / c_{k}\right]_{N}
$$


Hence, knowing all the cusps $\left[a_{j} / c_{j}\right]$ reduces the task of finding all possible poles to the inspection of $q$-expansions of $f$ at $a_{j} / c_{j}$; i.e., of $q$-expansions of $f\left(\gamma_{j} \tau\right)$ as in $(15.3)$ with $\gamma_{j} \in \mathrm{SL}_{2}(\mathbb{Z})$ such that $\gamma_{j} \infty=a_{j} / c_{j}$. We call these expansions also local $q$-expansions of $f^{*}$ at the cusps $\left[a_{j} / c_{j}\right]_{N} ; w_{N}\left(c_{j}\right)$ is called the width of the cusp $\left[a_{j} / c_{j}\right]_{N}$. It is straightforward to show that it is independent of the choice of the representative $a_{j} / c_{j}$ of the cusp $\left[a_{j} / c_{j}\right]_{N}$, and that $w_{N}\left(c_{j}\right)=N / \operatorname{gcd}\left(c_{j}^{2}, N\right)$ for relatively prime $a_{j}$ and $c_{j}$. Note that $[\infty]_{N}=[1 / 0]_{N}$.

Definition 15.3 (Order and $\phi$-order). Let $f=\sum_{n=m}^{\infty} a_{n} q^{n}$ with $m \in \mathbb{Z}$, such that $a_{m} \neq 0$. Then we define the order of $f$ as

$$
\text { ord } f:=m \text {. }
$$

More generally, if $\phi=\sum_{n=1}^{\infty} b_{n} q^{n / w}$ for some fixed $w \in \mathbb{Z}_{>0}$, and $F=f \circ \phi:=$ $\sum_{n=m}^{\infty} a_{n} \phi^{n}$, then we define the $\phi$-order of $f$ as

$$
\operatorname{ord}_{\phi} f:=m \text {. }
$$

(e.g., if $m=$ ord $f=-1$ and $\phi=q^{2}$, then $\operatorname{ord}_{\phi} F=-1$, but ord $F=-2$; if $m=\operatorname{ord} f=-2$ and $\phi=q^{1 / 2}$, then $\operatorname{ord}_{\phi} F=-2$, but ord $F=-1$.) In addition, more generally, we extend this definition of $\phi$-order to the case, where $\phi:=\phi_{\tau_{0}}(\tau)$ is one of the charts as in (9.1), (9.2), and (9.3).

The order $\operatorname{ord}_{[a / c]_{\ell}} f^{*}$ of $f^{*}$ at a cusp $[a / c]_{\ell}$ is defined to be the $q^{1 / w_{\ell}(c)_{-}}$ order of a local $q$-expansion of $f^{*}$ at $[a / c]_{N}$, that is,

$$
\operatorname{ord}_{[a / c]_{N}} f^{*}:=\operatorname{ord}_{q^{1 / w_{N}}(c)} f(\gamma \tau) \text { where } \gamma=\left(\begin{array}{ll}
a & b \\
c & d
\end{array}\right) \in \mathrm{SL}_{2}(\mathbb{Z}) .
$$

It is straightforward to verify that $\operatorname{ord}_{[a / c]} f^{*}$ is well-defined. For a concrete example, see Example 2.1.

\section{Appendix: Meromorphic Functions on Riemann Surfaces-Basic Notions}

To make this article as much self-contained as possible, in this second appendix section, we recall most of the facts that we need about meromorphic functions on Riemann surfaces. For the terminology, we basically follow [7]; other classic texts are [6] and [11].

Lemma 15.2 states the fundamental fact that any analytic function on a compact Riemann surface is constant. In Example 2.1, we have seen that $z_{5}^{*}$ has its only zero of order 1 at $[\infty]_{5}$ and its only pole at $[0]_{5}$ with multiplicity 1 , i.e., $z_{5}^{*}$ has order -1 at $[\infty]_{5}{ }^{16}$ This is also in accordance with Lemma 16.1 , a corollary of another fundamental fact which says that meromorphic functions on compact Riemann surfaces have exactly as many zeroes as poles (counting multiplicities); see, for instance, [11, Proposition 4.12]:

\footnotetext{
${ }^{16}$ Notice that we also say that $z_{5}^{*}$ has pole order 1 at $[\infty]_{5}$.
} 
Lemma 16.1. Let $g$ be a non-constant meromorphic function on a compact Riemann surface $X$. Then

$$
\sum_{x \in X} \operatorname{ord}_{x} g=0 .
$$

Here, $\operatorname{ord}_{x_{0}} g$ is defined as follows. Suppose $g(x)=\sum_{n=m}^{\infty} c_{n}(\varphi(x)-$ $\left.\varphi\left(x_{0}\right)\right)^{n}, c_{m} \neq 0$, is the local Laurent expansion of $g$ at $x_{0}$ using the local coordinate chart $\varphi: U_{0} \rightarrow \mathbb{C}$ which homeomorphically maps a neighborhood $U_{0}$ of $x_{0} \in X$ to an open set $V_{0} \subseteq \mathbb{C}$. Then, $\operatorname{ord}_{x_{0}} g:=m$.

Let $\mathcal{M}(S)$ denote the field of meromorphic functions $f: S \rightarrow \hat{\mathbb{C}}$ on a Riemann surface $S .{ }^{17}$ Let $f \in \mathcal{M}(S)$ be non-constant: then for every neighborhood $U$ of $x \in S$, there exist neighborhoods $U_{x} \subseteq U$ of $x$ and $V$ of $f(x)$, such that the set $f^{-1}(v) \cap U_{x}$ contains exactly $k$ elements for every $v \in V \backslash\{f(x)\}$. This number $k$ is called the multiplicity of $f$ at $x$; notation: $k=\operatorname{mult}_{x}(f){ }^{18}$ If $S$ is compact, $f \in \mathcal{M}(S)$ is surjective and each $v \in \hat{\mathbb{C}}$ has the same number of preimages, say $n$, counting multiplicities; i.e., $n=\sum_{x \in f^{-1}(v)} \operatorname{mult}_{x}(f)$, see, e.g., [7, Theorem 4.24]. This number $n$ is called the degree of $f$; notation: $n=\operatorname{Deg}(f)$. One of the consequences is that non-constant functions on compact Riemann surfaces have as many (finitely many) zeros as poles counting multiplicities; this is Lemma 16.1 .

$\operatorname{RamiPts}(f):=\left\{x \in S: \operatorname{mult}_{x}(f) \geq 2\right\}$ denotes the set of ramification points of $f$; $\operatorname{BranchPts}(f):=f(\operatorname{RamiPts}(f)) \subseteq \hat{\mathbb{C}}$ denotes the set of branch points of $f$. Ramification points, and also branch points, of a function $f$ form sets having no accumulation point. Hence, for functions on compact Riemann surfaces, these sets have finitely many elements.

\section{Conclusion}

In this article, we present the first proof of the Weierstraß gap theorem (for modular functions) without using the Riemann-Roch theorem. The main ingredient in our proof is the concept of order-reduction polynomials which corresponds to the discriminant of a field extension of $\mathbb{Q}$ in the setting of algebraic number theory, see, for instance, [10, III, §3]. In the field case, the structure of this discriminant is related to the ramification index [10, III, §2, Proposition 8, and III, §3, Proposition 14]. Analogously, in Proposition 11.5, we give a factorization of the order-reduction polynomial which in direct fashion relates to the branch points of the modular function $t$. This relation allows us to connect the degree of this polynomial to the genus of $X_{0}(N)$. This observation is crucial for our proof of the Weierstraß gap theorem.

In addition, our approach gives new algebraic and algorithmic insight based on module presentations of modular function algebras, in particular, the usage of integral bases. For example, our proof also gives a method to

\footnotetext{
${ }^{17}$ In this context, $\hat{\mathbb{C}}:=\mathbb{C} \cup\{\infty\}$ is understood to be a compact Riemann surface isomorphic to the Riemann sphere.

${ }^{18}$ If $x$ is a pole of $f: \operatorname{mult}_{x} f=-\operatorname{ord}_{x} f$; otherwise, $\operatorname{mult}_{x} f=\operatorname{ord}_{x}(f-f(x))$.
} 
compute the order-reduction polynomial by using the Puiseux series expansions at infinity. Another new feature concerns the gap bound: the main task of our proof is to show that there are exactly $g$ gaps for any modular function algebra. The proof that the corresponding pole orders are bounded by $2 g-1$, with the help of an elementary combinatorial argument turns out to be an immediate consequence of our approach. Another by-product of our framework is a natural explanation of the genus $g=0$ case as a consequence of the reduction to an integral basis.

Summarizing, our setting generalizes ideas from algebraic number theory, but still stays close to "first principles." Hence, we feel that our approach has potential for further extensions and applications. For example, we are planning to exploit the algorithmic content of our approach for computer algebra applications, for instance, for the effective computation of suitable module bases for modular function algebras.

\section{Acknowledgements}

Open access funding provided by Johannes Kepler University Linz. Both authors were supported by grant SFB F50-06 of the Austrian Science Fund (FWF). In November 2018, while working on parts of this paper, the first named author enjoyed the overwhelming hospitality of Bill Chen and his team at the Center for Applied Mathematics, Tianjin University. Finally, we thank Krishnaswami Alladi and an anonymous referee for constructive remarks which helped to improve the presentation of this paper.

Open Access. This article is distributed under the terms of the Creative Commons Attribution 4.0 International License (http://creativecommons.org/licenses/ by/4.0/), which permits unrestricted use, distribution, and reproduction in any medium, provided you give appropriate credit to the original author(s) and the source, provide a link to the Creative Commons license, and indicate if changes were made.

Publisher's Note Springer Nature remains neutral with regard to jurisdictional claims in published maps and institutional affiliations.

\section{References}

[1] Cohen, H., Strömberg, F.: Modular Forms: a Classical Approach. Graduate Studies in Mathematics, 179. American Mathematical Society, Providence, RI (2017)

[2] Dedekind, R., Weber, H.: Theory of Algebraic Functions of One Variable. Translated and introduced by John Stillwell. History of Mathematics, 39. American Mathematical Society, Providence, RI (2012)

[3] Dedekind, R., Weber, H.: Theorie der algebraischen Functionen einer Veränderlichen. J. Reine Angew. Math. 92, 181-290 (1882) 
[4] Del Centina, A.: Weierstrass points and their impact in the study of algebraic curves: a historical account from the "Lückensatz" to the 1970s. Ann. Univ. Ferrara Sez. VII Sci. Mat. 54(1), 37-59 (2008)

[5] Diamond, F., Shurman, J.: A First Course in Modular Forms. Graduate Texts in Mathematics, 228. Springer-Verlag, New York (2005)

[6] Farkas, H.M., Kra, I.: Riemann Surfaces. Graduate Texts in Mathematics, 71. Springer-Verlag, New York-Berlin (1980)

[7] Forster, O.: Lectures on Riemann Surfaces. Graduate Texts in Mathematics, 81. Springer-Verlag, New York-Berlin (1981)

[8] Graham, R.L., Knuth, D.E., Patashnik, O.: Concrete Mathematics: a Foundation for Computer Science. Second Edition. Addison-WesleyPublishing Company, Reading, MA (1994)

[9] Knopp, M.I.: Modular Functions in Analytic Number Theory. American Mathematical Society, Providence, RI (1993)

[10] Lang, S.: Algebraic Number Theory. Second Edition. Graduate Texts in Mathematics, 110. Springer-Verlag, New York (1994)

[11] Miranda, R.: Algebraic Curves and Riemann Surfaces. Graduate Studies in Mathematics, 5. American Mathematical Society, Providence, RI (1995)

[12] Ono, K.: The Web of Modularity: Arithmetic of the Coefficients of Modular Forms and $q$-Series. CBMS Regional Conference Series in Mathematics, 102. American Mathematical Society, Providence, RI (2004)

[13] Paule, P., Radu, C.-S.: A new witness identity for $11 \mid p(11 n+6)$. In: Andrews, G.E., Garvan, F. (eds.) Analytic Number Theory, Modular Forms and q-Hypergeometric Series, pp. 625-639. Springer Proc. Math. Stat., 221, Springer, Cham (2017)

[14] Paule, P., Radu, C.-S.: A unified algorithmic framework for Ramanujan's congruences modulo powers of 5, 7, and 11. Submitted (2018)

[15] Radu, C.-S.: An algorithmic approach to Ramanujan-Kolberg identities. J. Symbolic Comput. 68(1), 225-253 (2015)

[16] Radu, C.-S.: An algorithm to prove algebraic relations involving eta quotients. Ann. Comb. 22(2), 377-391 (2018)

Peter Paule and Cristian-Silviu Radu

Research Institute for Symbolic Computation (RISC)

Johannes Kepler University

4040 Linz

Austria

e-mail: Silviu.Radu@risc.uni-linz.ac.at 


\section{Peter Paule}

e-mail: Peter.Paule@risc.uni-linz.ac.at

Received: 31 January 2019.

Accepted: 7 August 2019. 